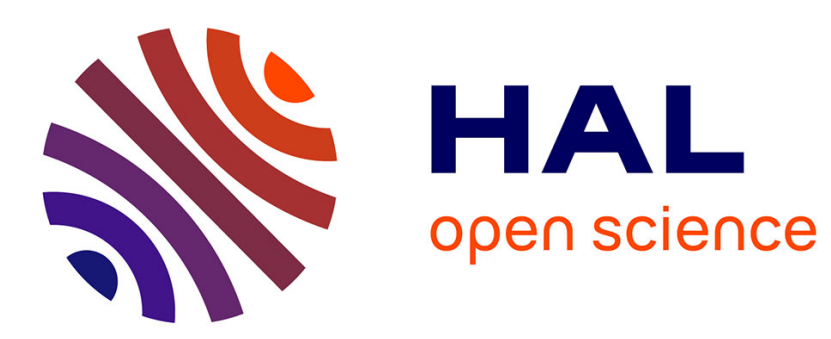

\title{
Chance-constrained programming with fuzzy stochastic coefficients
}

\author{
Farid Aiche, Moncef Abbas, Didier Dubois
}

\section{To cite this version:}

Farid Aiche, Moncef Abbas, Didier Dubois. Chance-constrained programming with fuzzy stochastic coefficients. Fuzzy Optimization and Decision Making, 2013, 12 (2), pp.125-152. 10.1007/s10700-0129151-8 . hal-01121983

\section{HAL Id: hal-01121983 \\ https://hal.science/hal-01121983}

Submitted on 3 Mar 2015

HAL is a multi-disciplinary open access archive for the deposit and dissemination of scientific research documents, whether they are published or not. The documents may come from teaching and research institutions in France or abroad, or from public or private research centers.
L'archive ouverte pluridisciplinaire HAL, est destinée au dépôt et à la diffusion de documents scientifiques de niveau recherche, publiés ou non, émanant des établissements d'enseignement et de recherche français ou étrangers, des laboratoires publics ou privés. 


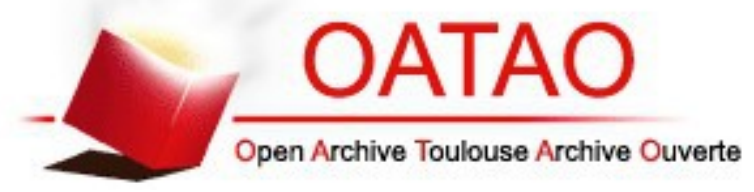

\section{Open Archive TOULOUSE Archive Ouverte (OATAO)}

OATAO is an open access repository that collects the work of Toulouse researchers and makes it freely available over the web where possible.

This is an author-deposited version published in : http://oatao.univ-toulouse.fr/ Eprints ID : 12527

To link to this article : DOI :10.1007/s10700-012-9151-8

URL : http://dx.doi.org/10.1007/s10700-012-9151-8

To cite this version : Aiche, Farid and Abbas, Moncef and Dubois,

Didier Chance-constrained programming with fuzzy stochastic

coefficients. (2013) Fuzzy Optimisation and Decision-Making, vol. 12

(n²). pp. 125-152. ISSN 1568-4539

Any correspondance concerning this service should be sent to the repository administrator: staff-oatao@,listes-diff.inp-toulouse.fr 


\title{
Chance-constrained programming with fuzzy stochastic coefficients
}

\author{
Farid Aiche • Moncef Abbas • Didier Dubois
}

\begin{abstract}
We consider fuzzy stochastic programming problems with a crisp objective function and linear constraints whose coefficients are fuzzy random variables, in particular of type $L-R$. To solve this type of problems, we formulate deterministic counterparts of chance-constrained programming with fuzzy stochastic coefficients, by combining constraints on probability of satisfying constraints, as well as their possibility and necessity. We discuss the possible indices for comparing fuzzy quantities by putting together interval orders and statistical preference. We study the convexity of the set of feasible solutions under various assumptions. We also consider the case where fuzzy intervals are viewed as consonant random intervals. The particular cases of type $L-R$ fuzzy Gaussian and discrete random variables are detailed.
\end{abstract}

Keywords Fuzzy random variables · Fuzzy intervals - Random intervals . Convexity $\cdot$ Fuzzy stochastic program $\cdot$ Probability $\cdot$ Possibility $\cdot$ Necessity

\section{Introduction}

The chance-constrained programming method was first introduced by Charnes and Cooper (1959). The idea was to model linear constraints with random coefficients

\author{
F. Aiche \\ Université Mouloud Mammeri, BP 17 RP, Tizi-ouzou, Algeria \\ M. Abbas \\ Faculté de Mathématiques, USTHB, LAID3, BP 32 EL, 16311 Alia, Alger, Algeria \\ D. Dubois $(\varangle)$ \\ IRIT, CNRS and University of Toulouse, 118, Route de Narbonne, \\ 31062 Toulouse Cedex 9, France \\ e-mail: dubois@irit.fr
}


so that their solutions have a sufficiently high probability of being feasible. This formulation makes it possible to convert stochastic constraints into equivalent deterministic ones. This technique has had in the last years several applications such as the $\mathrm{P}$-model or minimum risk model, which consists in maximising the probability that some objective function is attained at least to a predetermined level. A fuzzy counterpart to chance-constrained programming, namely linear programming with constraints having fuzzy interval coefficients was proposed by Dubois (1987) and later studied by others such as Inuiguchi et al. (1992), Inuiguchi and Ramik (2000), where probability is replaced by possibility or necessity. While the chance-constrained framework aims at finding solutions valid most of the time, the handling of fuzzy data relies on the decision-maker's attitude in front of ambiguity: for instance, necessity dominance indices try to achieve robustness in front of partial information, a pessimistic attitude trying to find good solutions that are relevant despite the lack of precision of the data (Dubois et al. 2001).

However, in practice, we may be faced with situations where, at the same time, coefficients in an optimisation problem are random variables and their realisations are not completely known. This is the case when the optimisation problem coefficients cover a set of possible scenarios (expressing variability of situations where an optimal decision is to be made), each of which is imprecisely known (for instance, precision of measured values is limited). When random variables take values that are known through fuzzy intervals, it leads to the concept of fuzzy random variables, first introduced by Kwakernaak (1978). Later, other authors like Kruse and Meyer (1987), Puri and Ralescu (1986), among others studied this concept. Puri and Ralescu consider a fuzzy random variable as a classical one taking values on a space of fuzzy sets understood as a metric space of membership functions. Kwakernaak, as well as Kruse and Meyer, consider a fuzzy random variable as a function from a probability space to a set of fuzzy intervals, where the latter restrict the actual values of standard random variables. This is the view adopted here. Recently Couso and Dubois (2009), Couso and Sánchez (2011) proposed yet another interpretation of this concept as a conditional possibility measure dominating a set of conditional probabilities, and they compare it to the two other views.

There exist a number of past works addressing fuzzy and probabilistic features conjointly in optimisation problems (Chakraborty et al. 1994; Yazini 1987; Qiao and Wang 1993; Qiao et al. 1994; Wang and Qiao 1993). In fact there are papers dealing with fuzzy random objective functions (Li et al. 2006; Katagiri et al. 2008; Qiao and Wang 1993; Qiao et al. 1994; Wang and Qiao 1993) and papers dealing with fuzzy random coefficients in constraints (Qiao and Wang 1993; Qiao et al. 1994; Wang and Qiao 1993; Aiche 1995; Luhandjula 1996). This paper focuses on the latter problem, and more specifically on various ways of turning fuzzy random constraints into deterministic counterparts. Wang and Qiao (1993) study a formulation of multiobjective linear programming problems with fuzzy random coefficients in the objective and constraints. However they reduce the problem to standard stochastic programming by considering $\alpha$-cuts of fuzzy coefficients, and defining two sets of constraints, one using the upper bound of cuts and the other by means of lower bounds of cuts. This is only 
one possible way to go, but the systematic choice of lower or upper-bounds of cuts in both sides of the constraints is somewhat debatable. In Ammar (2009), recently studied a similar formulation of multiobjective linear programming problems with fuzzy random coefficients in the objective and constraints. Katagiri et al. (2004) handle fuzzy number comparisons in fuzzy random bottleneck optimisation using possibility and necessity of dominance. A similar formulation for multiobjective linear programming is proposed by $\mathrm{Li}$ et al. (2006). By nesting possibilistic programming inside chance-constrained programming, they transform the fuzzy stochastic constraints into equivalent deterministic ones. Likewise, Iskander (2005) used the standard chanceconstrained approach by transforming stochastic fuzzy problems in the presence of fuzzy coefficients and random variables into their deterministic equivalent according to the four possibilistic dominance indices introduced by Dubois and Prade (1983). To solve the general problem, in Aiche (1995) and Luhandjula (1996) a semi-infinite approach was proposed in order to convert it to a stochastic one which can solved by chance-constrained programming (Charnes and Cooper 1959) or a two-stage programming method (Dantzig 1955). Luhandjula (2004) proposed an approach to transform constraints in the presence of fuzzy random variables into deterministic constraints, by comparing intervals obtained from prescribed cuts of fuzzy coefficients. Luhandjula and Gupta (1996) generalize robust programming with interval coefficients to the fuzzy stochastic framework, turning equality constraint is into fuzzy inclusion constraints. These works are surveyed again in Luhandjula (2006). Luhandjula and Joubert (2010) further investigate optimisation models in a fuzzy stochastic environment and approaches to convert them into deterministic problems, focusing on the Gaussian case.

In this paper, we try to organise the possible formulations of random fuzzy constraints in a reasoned way. We consider fuzzy stochastic programming problems, with a precise objective function and linear constraints whose coefficients are represented by random variables whose values are known through fuzzy intervals, first in the general case, and then when coefficients are random fuzzy intervals of type $L-R$. We start by noticing that the comparison of fuzzy intervals can benefit from techniques that compare intervals and techniques that compare probabilities. This remark leads to revisit some known methods for comparing fuzzy intervals by combining these two basic techniques. We then discuss three versions of chance-constrained programming with fuzzy stochastic coefficients: (i) by combining probability and possibility, or probability and necessity; (ii) using probability over defuzzified fuzzy quantities; (iii) and by combining chance-constrained programming and random interval comparisons; in the latter case a fuzzy interval is viewed as a random interval. We also consider the particular case of fuzzy intervals of type $L-R$.

The paper is organised as follows. In the next section, variants of fuzzy random variables are briefly recalled. In Sect. 3, we recall methods for the comparison of random numbers, intervals, and fuzzy intervals. In Sect. 4, we present four versions of chance-constrained programming with fuzzy stochastic coefficients. The conditions of convexity of the feasible sets obtained via the various formulations are studied. In the appendix, we recall some relevant basic definitions and properties useful for the paper. 


\section{Fuzzy random variables}

Fuzzy random variables were first introduced by Kwakernaak (1978).

Definition 1 Let $(\Omega, F, P)$ be a probability space. A fuzzy random variable $\tilde{x}$ is a function $\Omega \rightarrow \mathcal{F}(\mathbb{R}): \omega \mapsto \tilde{x}(\omega)$ from $(\Omega, F, P)$ to a set of fuzzy intervals $\mathcal{F}(\mathbb{R})$.

Basic notions and notations for fuzzy intervals are provided in Appendix A. For $\tilde{x}(\omega)$ to be a fuzzy interval, we assume that its $\alpha$-cuts are closed intervals $\tilde{x}^{\alpha}(\omega)=$ $\left[\underline{x}^{\alpha}(\omega), \bar{x}^{\alpha}(\omega)\right]$, for $0<\alpha \leq 1$, where $\underline{x}^{\alpha}(\omega)=\inf \left\{x \in \mathbb{R}: \mu_{\tilde{x}(\omega)}(x) \geq \alpha\right\}, \bar{x}^{\alpha}(\omega)=$ $\sup \left\{x \in \mathbb{R}: \mu_{\tilde{x}(\omega)}(x) \geq \alpha\right\}, \alpha>0$, and $\mu_{\tilde{x}(\omega)}(x)$ is the membership degree of $x \in \tilde{x}(\omega)$.

Kwakernaak (1978), as well as Kruse and Meyer later on, consider that a fuzzy random variable $\tilde{x}$ describes the vague perception of a crisp unobservable original random variable $x$. In their view, the degree of membership of a standard random variable $x: \Omega \rightarrow \mathbb{R}$ to $\tilde{x}$ is computed as $\mu_{\tilde{x}}(x)=\inf _{\omega \in \Omega} \mu_{\tilde{x}(\omega)}(x(\omega))$. It represents the degree of possibility that the random variable $x$ is a representative of $\tilde{x}$.

In what follows, we restrict to special cases of fuzzy random variables, that are often used in practice.

1. Discrete fuzzy random variables: Let $\Omega=\left\{\omega_{1}, \ldots, \omega_{r}\right\}$ be a finite probability space, equipped with a discrete probability distribution $P\left(\omega_{k}\right)=q_{k}, k=$ $1,2, \ldots, r$ and $\sum_{k=1}^{k=r} q_{k}=1$. A discrete fuzzy random variable $\tilde{x}$ is a fuzzy random variable, each random realization of which is a fuzzy interval $\tilde{a}_{k}$ having a positive probability of being the observed perception, that is, $P\left(\tilde{x}\left(\omega_{k}\right)=\tilde{a}_{k}\right)=$ $q_{k}, k=1,2, \ldots, r$, where $\tilde{a}_{k}, k=1,2, \ldots, r$ are fuzzy intervals.

2. Normal fuzzy random variables: Based on the view of Kwakernaak (1978), we consider an original normally distributed random variable $x$ with a crisp mean $\mu$ and a precise variance $\sigma^{2}$. In practice, the mean $\mu$ of $x$ may not be completely known; it is represented by fuzzy interval $\tilde{\mu}$, which represents "around $\mu$ ". Then, following Shapiro (2009), we consider a fuzzy random variable $\tilde{x}$ that is normally distributed with a fuzzy mean $\tilde{\mu}$ and a precise variance $\sigma^{2}$. For each $\alpha$-cut $\tilde{\mu}^{\alpha}=\left[\mu^{\alpha}, \bar{\mu}^{\alpha}\right]$ of $\tilde{\mu}$, note that $\underline{x}^{\alpha}$ and $\bar{x}^{\alpha}$ are crisp normal random variables with corresponding means $\mu^{\alpha}$ and $\bar{\mu}^{\alpha}$ and precise variance $\sigma^{2}$. Since $\mu^{\alpha} \leq \mu \leq \bar{\mu}^{\alpha}$, it follows that $\forall t \in \mathbb{R}, F_{\underline{x}^{\alpha}}(t) \leq F_{x}(t) \leq F_{\bar{x}^{\alpha}}(t)$, where $F_{\underline{x}^{\alpha}}, \bar{F}_{x}$ and $F_{\bar{x}^{\alpha}}$ are cumulative distribution functions of $\underline{x}^{\alpha}, x$ and $\bar{x}^{\alpha}$, respectively.

For details see Shapiro (2009).

3. Fuzzy random variables of type $L-R$ : Let $\mathcal{F}_{L R}(\mathbb{R})$ be the set of fuzzy intervals $\tilde{a}=\left(\underline{a}, \bar{a}, \delta^{a}, \gamma^{a}\right)$ of type $L-R$ (Dubois and Prade 1988). Their $\alpha$-cuts are of the form $\left[\underline{a}-L^{-1}(\alpha) \delta^{a}, \bar{a}+R^{-1}(\alpha) \gamma^{a}\right]$, where $\alpha \in(0,1]$, the shape functions $L$ and $R$ are defined on the positive real line $[0, \infty)$, non-negative, non-increasing, and upper semi-continuous, such that $L(0)=R(0)=1$, and $\delta^{a}, \gamma^{a}$ are positive real numbers and represent, respectively the left and right spreads of $\tilde{a}$. Moreover, $L^{-1}(\alpha)=\sup \{s: L(\alpha) \geq s\}$ and $R^{-1}(\alpha)=\sup \{s: R(\alpha) \geq s\}$. Replacing $\mathcal{F}(\mathbb{R})$ by $\mathcal{F}_{L R}(\mathbb{R})$ in the previous definition, then $\tilde{x}$ is called fuzzy random variable of type $L-R$ and its realizations denoted by $\tilde{x}(\omega)=\left(\underline{x}(\omega), \bar{x}(\omega), \delta^{x}, \gamma^{x}\right)$. In other words $\tilde{x}^{\alpha}(\omega)=\left[\underline{x}(\omega)-L^{-1}(\alpha) \delta^{x}, \bar{x}(\omega)+R^{-1}(\alpha) \gamma^{x}\right]$. 
4. Normal fuzzy random variables of type $L-R: \tilde{x}(\omega)=\left(\underline{x}(\omega), \bar{x}(\omega), \delta^{x}, \gamma^{x}\right)$ is a normal fuzzy random variable of type $L-R$ with fuzzy mean $\tilde{\mu}=\left(\mu, \bar{\mu}, \delta^{x}, \gamma^{x}\right)$, which is another fuzzy interval of type $L-R$, and precise variance $\sigma^{2}$. Then, $\underline{x}(\omega)$ and $\bar{x}(\omega)$ (with $\underline{x} \leq \bar{x}$ ) are normal random variables with the corresponding means $\mu, \bar{\mu}$ and the same precise variance $\sigma^{2}$.

5. Discrete fuzzy random variables of type $L-R: \tilde{x}(\omega)=\left(\underline{x}(\omega), \bar{x}(\omega), \delta^{x}, \gamma^{x}\right)$ is a discrete fuzzy random variable of type $L-R$ and $\underline{x}(\omega)$ and $\bar{x}(\omega)$ (with $\underline{x} \leq \bar{x}$ ) are discrete random variables.

In this paper, we consider fuzzy stochastic programming problems with a deterministic objective function and linear constraints where coefficients are fuzzy random variables, in particular of type $L-R$, as follows:

$$
\left(P_{F S}\right)\left\{\begin{array}{l}
\max \phi(x) \\
\sum_{j=1}^{n} \tilde{a}_{i j}(\omega) \odot x_{j} \leq \tilde{b}_{i}(\omega), i=1, \ldots, m \\
x_{j} \geq 0, j=1, \ldots, n
\end{array}\right.
$$

where $\phi(x)$ is a deterministic objective function, $\tilde{a}_{i j}$ and $\tilde{b}_{i}$ are fuzzy random variables. And $\sum_{j=1}^{n}, \odot$ denote the generalization of, respectively addition and multiplication by means of the extension principle (see Appendix A for basic definitions). Moreover, $\leq$ refers to suitable extensions of the inequality between real numbers to fuzzy intervals (Dubois and Prade 1988, 1987a). The contribution of this paper is to survey various approaches to express such inequality constraints for random fuzzy coefficients of linear expressions. The use of the chance-constrained framework enables deterministic counterparts of these fuzzy stochastic constraints to be formulated.

\section{Comparing uncertain quantities}

In order to compare linear expressions that take the form of fuzzy random variables, one must be in a position to compare intervals, fuzzy intervals and random numbers. Moreover fuzzy intervals can also be interpreted as nested random intervals (Dubois and Prade 1987b).

\subsection{Comparing intervals}

Let $[\underline{a}, \bar{a}]$ and $[\underline{b}, \bar{b}]$ be two intervals. Comparing the intervals $[\underline{a}, \bar{a}]$ and $[\underline{b}, \bar{b}]$, we can choose between four basic order relations $\geq_{i}, i=1, \ldots, 4$, as follows:

1. $[\underline{a}, \bar{a}] \geq 1[\underline{b}, \bar{b}] \Leftrightarrow \underline{a} \geq \bar{b}$

2. $[\underline{a}, \bar{a}] \geq_{2}[\underline{b}, \bar{b}] \Leftrightarrow \underline{a} \geq \underline{b}$

3. $[\underline{a}, \bar{a}] \geq_{3}[\underline{b}, \bar{b}] \Leftrightarrow \bar{a} \geq \bar{b}$

4. $[\underline{a}, \bar{a}] \geq_{4}[\underline{b}, \bar{b}] \Leftrightarrow \bar{a} \geq \underline{b}$.

As usual $>_{i}$ denotes the strict part of $\geq_{i}$. The relation $\geq_{1}$ is the most demanding, $\geq_{4}$ is the least demanding, $\geq_{2}$ and $\geq_{3}$ are of intermediary strength. In fact, if $[\underline{a}, \bar{a}]$ models 
an ill-known value $x$ and $[\underline{b}, \bar{b}]$ an ill-known quantity $y, x \geq_{1} y$ is a robust inequality since it holds whatever the values of $x$ and $y$ are; $x \geq_{2} y$ expresses a pessimistic attitude (if the higher $x$ and $y$, the better); $x \geq_{3} y$ expresses an optimistic attitude; while $x \geq_{4} y$ expresses an adventurous attitude, since it may well be that $y>x$ when their values are eventually known.

These relations are known in the literature:

- The strict relation $>_{1}$ is known to be an interval order (Fishburn 1987), and $[\underline{a}, \bar{a}]>_{1}[\underline{b}, \bar{b}] \Leftrightarrow \neg\left([\underline{b}, \bar{b}] \geq_{4}[\underline{a}, \bar{a}]\right)$.

- The simultaneous use of $\geq_{2}$ and $\geq_{3}$ :

$$
[\underline{a}, \bar{a}] \succeq[\underline{b}, \bar{b}] \text { if and only if }[\underline{a}, \bar{a}] \geq_{2}[\underline{b}, \bar{b}] \text { and }[\underline{a}, \bar{a}] \geq_{3}[\underline{b}, \bar{b}]
$$

is the canonical order induced by the lattice structure of intervals, equipped with the operations max and min extended to intervals $(\max ([\underline{a}, \bar{a}],[\underline{b}, \bar{b}])=$ $[\max (\underline{a}, \underline{b}), \max (\bar{a}, \bar{b})]$, and likewise for $\min )$ :

$$
\begin{aligned}
{[\underline{a}, \bar{a}] \succeq[\underline{b}, \bar{b}] } & \Longleftrightarrow \max ([\underline{a}, \bar{a}],[\underline{b}, \bar{b}])=[\underline{a}, \bar{a}] \\
& \Longleftrightarrow \min ([\underline{a}, \bar{a}],[\underline{b}, \bar{b}])=[\underline{b}, \bar{b}] .
\end{aligned}
$$

We call it lattice interval order.

It makes sense to use the latter ordering when comparing non-independent quantities $x$ and $y$. For instance, if $x$ and $y$ depend on a parameter $\lambda$, so that $x=\lambda \underline{a}+(1-\lambda) \bar{a}$ and $y=\lambda \underline{b}+(1-\lambda) \bar{b}$, then $x>y, \forall \lambda$ implies $x \succeq y$, not $x>_{1} y$.

\subsection{Statistical preference}

Statistical preference measures the probability that a random variable $a$ is greater than another one $b$, as $P(a>b)=P\left(\left\{\left(\omega, \omega^{\prime}\right): a(\omega)>b\left(\omega^{\prime}\right)\right\}\right)$ (David 1963). One of the two following opposite assumptions is often made:

- independent random variables with continuous density functions $p_{a}$ and $p_{b}$ : then $P(a>b)=\int_{x>y} p_{a}(x) p_{b}(y) d x d y$. In the case of independent random variables $a$ and $b, P(a>b)=1$ is generally equivalent to $\operatorname{Support}(a)>{ }_{1} \operatorname{Support}(b)$.

- comonotone random variables with a functional link of the form $\omega=\omega^{\prime}$ : then $P(a>b)=P(\{\omega: a(\omega)>b(\omega)\})$.

Then define $a \geq_{\alpha}^{P} b \Longleftrightarrow P(a \geq b)>\alpha$. For $\alpha>\frac{1}{2}$, this is the kind of dominance used in chance-constrained programming.

Another way of handling probabilistic constraints is to replace random coefficients by their expectations. But this method is sometimes an oversimplification of the real problem and its solution is not always easy to interpret (it is not clear it always yields the best solution in the average). 


\subsection{Comparing fuzzy intervals}

There are several methods for comparing fuzzy intervals (Wang and Kerre 2001). Many were proposed in a rather ad hoc way. Here we consider three approaches, according to whether fuzzy intervals are viewed as possibility distributions, or as nested random intervals, or yet are defuzzified. These approaches extend or combine in some way interval comparisons and statistical preference.

\subsubsection{Possibilistic preference}

Consider two fuzzy intervals $\tilde{a}$ and $\tilde{b}$ with membership functions $\mu_{\tilde{a}}$ and $\mu_{\tilde{b}}$, respectively. In what follows the abbreviation pos and nec represent, respectively possibility and necessity (Dubois and Prade 1988). The possibility and necessity of preference of $\tilde{a}$ over $\tilde{b}$, denoted, respectively by $\operatorname{pos}(\tilde{a} \geq \tilde{b})$ and $\operatorname{nec}(\tilde{a}>\tilde{b})$ are defined as follows [see for instance (Dubois and Prade 1988, 1987a)]

$$
\begin{aligned}
& \operatorname{pos}(\tilde{a} \geq \tilde{b})=\sup _{x \geq y}\left(\min \left(\mu_{\tilde{a}}(x), \mu_{\tilde{b}}(y)\right)\right. \\
& \operatorname{nec}(\tilde{a}>\tilde{b})=1-\operatorname{pos}(\tilde{b} \geq \tilde{a})=1-\sup _{x \leq y}\left(\min \left(\mu_{\tilde{a}}(x), \mu_{\tilde{b}}(y)\right) .\right.
\end{aligned}
$$

The first of these indices was already proposed by Baas and Kwakernaak (1977). This approach is the natural counterpart to statistical preference in possibility theory; yet it is also an extension of interval-related orderings since it is easy to check, if the supports of $\tilde{a}$ and $\tilde{b}$ are bounded and their membership functions $\mu_{\tilde{a}}$ and $\mu_{\tilde{b}}$ are upper semi-continuous, that it comes down to comparing $\alpha$-cut intervals $\tilde{a}^{\alpha}$ and $\tilde{b}^{\alpha}$ using $\geq_{4}$, and $\tilde{a}^{1-\alpha}$ and $\tilde{b}^{1-\alpha}$ using $\geq_{1}$, respectively (Dubois 1987):

Proposition 1 If $\tilde{a}$ and $\tilde{b}$ are fuzzy intervals, the following equivalences hold:

- $\forall \alpha>0, \operatorname{pos}(\tilde{a} \geq \tilde{b}) \geq \alpha \Longleftrightarrow \bar{a}^{\alpha} \geq \underline{b}^{\alpha} \Longleftrightarrow \tilde{a}^{\alpha} \geq_{4} \tilde{b}^{\alpha}$,

- $\forall \alpha<1, \operatorname{nec}(\tilde{a}>\tilde{b})>\alpha \Longleftrightarrow \underline{a}^{1-\alpha}>\bar{b}^{1-\alpha} \Longleftrightarrow \tilde{a}^{1-\alpha}>{ }_{1} \tilde{b}^{1-\alpha}$.

Proof The first item is obvious. For the second item, $\operatorname{nec}(\tilde{a}>\tilde{b})>\alpha \Longleftrightarrow \operatorname{pos}(\tilde{b} \geq$ $\tilde{a})<1-\alpha$. That is $\sup _{y \geq x} \min \left(\mu_{\tilde{a}}(x), \mu_{\tilde{b}}(y)\right)<1-\alpha$. Then clearly this is equivalent to $\tilde{a}^{1-\alpha} \cap \tilde{b}^{1-\alpha}=\emptyset$. As $\operatorname{pos}(\tilde{a} \geq \tilde{b})=1, \tilde{a}^{1-\alpha}$ is on the right hand side of $\tilde{b}^{1-\alpha}$. As we deal with closed intervals, it follows that $\tilde{a}^{1-\alpha} \cap \tilde{b}^{1-\alpha}=\emptyset$ is equivalent to $\tilde{a}^{1-\alpha} \subset\left[\bar{b}^{1-\alpha},+\infty\right)$, and $\underline{a}^{1-\alpha} \neq \bar{b}^{1-\alpha}$. Hence $\operatorname{nec}(\tilde{a}>\tilde{b})>\alpha$ is equivalent to $\underline{a}^{1-\alpha}>\bar{b}^{1-\alpha}$.

N.B. The case when $\operatorname{nec}(\tilde{a}>\tilde{b})=1$ (or equivalently, $\operatorname{pos}(\tilde{b} \geq \tilde{a})=0$ ) is special, as its equivalent formulation in terms of interval ordering depends on the continuity properties of the membership function. If the support of $\tilde{a}$ and $\tilde{b}$ are closed intervals (for instance, if $\tilde{a}$ and $\tilde{b}$ are closed intervals), nec $(\tilde{a}>\tilde{b})=1$ means $\inf S(\tilde{a})>\sup S(\tilde{b})$, i.e., $S(\tilde{a})>1 S(\tilde{b})$, and the two supports are disjoint. If on the contrary, the membership functions are surjective on the unit interval and continuous, the supports are open intervals, e.g., $S(\tilde{a})=] \underline{a}^{0}, \bar{a}^{0}\left[\right.$, where $\underline{a}^{0}=\lim _{\alpha \rightarrow 0} \underline{a}^{\alpha}$ and $\bar{a}^{0}=\lim _{\alpha \rightarrow 0} \bar{a}^{\alpha}$. As a 
consequence, $\operatorname{nec}(\tilde{a}>\tilde{b})=1 \Longleftrightarrow \operatorname{pos}(\tilde{b} \geq \tilde{a})=0 \Longleftrightarrow \underline{a}^{0} \geq \bar{b}^{0}$. See Dubois and Prade (1983) for more details on pathological situations.

To generalize other relations $\geq_{2}, \geq_{3}$ to fuzzy intervals, we first interpret them as follows in the case of intervals:

$[\underline{a}, \bar{a}] \geq_{2}[\underline{b}, \bar{b}]: \forall x \in[\underline{a}, \bar{a}], \exists y \in[\underline{b}, \bar{b}]: x \geq y$, which encodes $\underline{a} \geq \underline{b} ;$

$[\underline{a}, \bar{a}] \geq 3[\underline{b}, \bar{b}]: \exists y \in[\underline{b}, \bar{b}], \forall x \in[\underline{a}, \bar{a}]: x \geq y$, which encodes $\overline{\bar{a}} \geq \overline{\bar{b}}$

(for $[\underline{a}, \bar{a}] \geq_{1}[\underline{b}, \bar{b}]$ and $[\underline{a}, \bar{a}] \geq_{4}[\underline{a}, \bar{a}]$, we use $\forall$ twice, and $\exists$ twice, respectively). The gradual extensions of these relations are then Dubois and Prade (1983):

$$
\begin{aligned}
& n \operatorname{nc}_{2}(\tilde{a} \geq \tilde{b})=\inf _{x} \max \left(1-\mu_{\tilde{a}}(x), \sup _{x \geq y} \mu_{\tilde{b}}(y)\right) \\
& \operatorname{pos}_{3}(\tilde{a}>\tilde{b})=\sup _{x} \min \left(\mu_{\tilde{a}}(x), \inf _{y \geq x} 1-\mu_{\tilde{b}}(y)\right) .
\end{aligned}
$$

Note that $\sup _{y: x \geq y} \mu_{\tilde{b}}(y)=\Pi((-\infty, x])$ (upper cumulative distribution), which is $\mu_{\tilde{b}}(x)$ if $x \leq \underline{b}^{1}$, and 1 otherwise. This fuzzy set can be denoted by $[\tilde{b},+\infty)$, and $n e c_{2}(\tilde{a} \geq \tilde{b})$ is the degree of inclusion of $\tilde{a}$ in $[\tilde{b},+\infty)$. Likewise, $\inf _{x \leq y} 1-\mu_{\tilde{b}}(y)=$ $N\left(\left(-\infty, x\right.\right.$ [) (lower cumulative distribution), which is $1-\mu_{\tilde{b}}(x)$ if $x \geq \bar{b}^{1}$, and 0 otherwise. This fuzzy set can be denoted by $] \tilde{b},+\infty)$; it is lower semi-continuous if $\tilde{b}$ is u.s.c. Then, $\operatorname{pos}_{3}(\tilde{a}>\tilde{b})$ is the degree of intersection of $\tilde{a}$ and $\left.] \tilde{b},+\infty\right)$.

And, as expected, if the supports of $\tilde{a}$ and $\tilde{b}$ are bounded and their membership functions $\mu_{\tilde{a}}$ and $\mu_{\tilde{b}}$ are upper semi-continuous,

Proposition 2 The following equivalences hold if $\mu_{\tilde{a}}$ and $\mu_{\tilde{b}}$ are upper semi-continuous, with no flat parts but for their cores:

- $\operatorname{nec} 2(\tilde{a} \geq \tilde{b}) \geq \alpha>0 \Longleftrightarrow \underline{a}^{1-\alpha} \geq \underline{b}^{\alpha} \Longleftrightarrow \tilde{a}^{1-\alpha} \geq_{2} \tilde{b}^{\alpha}$

- $\operatorname{pos}_{3}(\tilde{a}>\tilde{b}) \geq \alpha>0 \Longleftrightarrow \bar{a}^{\alpha} \geq \bar{b}^{1-\alpha} \Longleftrightarrow \tilde{a}^{\alpha} \geq_{3} \tilde{b}^{1-\alpha}$

Proof We use the following straightforward result:

Lemma 1 For any two fuzzy sets $F, G$ on a referential $S$, $\inf _{s \in S} \max \left(1-\mu_{F}(s), \mu_{G}(s)\right)$ $\geq \alpha>0$ if and only if $F^{\overline{1-\alpha}} \subseteq G^{\alpha}$, where $F^{\overline{1-\alpha}}$ is the strong $1-\alpha$-cut of $F$.

Then, $n e c_{2}(\tilde{a} \geq \tilde{b}) \geq \alpha>0$ means that $\tilde{a}^{\overline{1-\alpha}} \subseteq[\tilde{b},+\infty)^{\alpha}$, which is the same as $\underline{a}^{1-\alpha} \geq \underline{b}^{\alpha}$, under the assumptions of the Proposition. Moreover $\operatorname{pos}_{3}(\tilde{a}>\tilde{b}) \geq \alpha>$ 0 reads $\left.\left.\tilde{a}^{\alpha} \cap\right] \tilde{b},+\infty\right)^{\alpha} \neq \emptyset$. Due to the u.s.c. assumption, $\left.] \tilde{b},+\infty\right)^{\alpha}=\left[\bar{b}^{1-\alpha},+\infty\right)$, hence $\bar{a}^{\alpha} \geq \bar{b}^{1-\alpha}$.

Note that, except for pathological situations described in Dubois and Prade (1983), equalities $n e c_{2}(\tilde{a} \geq \tilde{b})+n e c_{2}(\tilde{b} \geq \tilde{a})=1$ hold, as well as $\operatorname{pos}_{3}(\tilde{a} \geq \tilde{b})+\operatorname{pos}_{3}(\tilde{b} \geq$ $\tilde{a})=1$ hold (e.g., with continuous membership functions).

\subsubsection{Random interval comparisons of fuzzy intervals}

Some authors consider a fuzzy interval as a nested random interval (Dubois and Prade 1987b). Namely the $\alpha$-cut $\left[\underline{a}^{\alpha}, \bar{a}^{\alpha}\right]$ of a continuous fuzzy interval $\tilde{a}$ depends on a 
random variable $\xi$ on the unit interval, that we can assume uniform (Lebesgue measure $\lambda)$. One then considers a fuzzy interval as a mapping from $([0,1], \mathcal{B}, \lambda)$ to the set of closed intervals $\mathcal{I}(\mathbb{R}): \xi \in[0,1] \mapsto\left[\underline{a}^{\xi}, \bar{a}^{\xi}\right]$. More generally the end points of the interval can depend on different random variables $\xi$ and $\zeta$, and the random interval can be of the form $\left[a^{\xi}, \bar{a}^{\zeta}\right]$ (Chanas and Nowakowski 1988).

Chanas et al. (1993), Chanas and Zielinski (1999) thus conjointly use interval comparisons and statistical preference for the comparison of fuzzy intervals. Namely, they generalize interval comparisons based on order relations $>_{i}, i=1,2,3,4$ to fuzzy intervals $\tilde{a}$ and $\tilde{b}$ understood as above.

1. $\mu_{1}(\tilde{a}, \tilde{b})=P\left(a^{\xi}>\bar{b}^{\zeta}\right)$

2. $\mu_{2}(\tilde{a}, \tilde{b})=P\left(\underline{a}^{\xi}>\underline{b}^{\zeta}\right)$

3. $\mu_{3}(\tilde{a}, \tilde{b})=P\left(\bar{a}^{\xi}>\bar{b}^{\zeta}\right)$

4. $\mu_{4}(\tilde{a}, \tilde{b})=P\left(\bar{a}^{\xi}>\underline{b}^{\zeta}\right)$

This is just the application of definitions proposed in the previous section for random intervals; $\xi$ and $\zeta$ could be independent, comonotonic or coupled by any copula. The actual form of $\mu_{i}$ depends on this copula. Two assumptions are considered by Chanas et al. (1993), Chanas and Zielinski (1999): functionally dependent fuzzy intervals and independent fuzzy intervals. Namely in the above four relations, they assume either $\xi=\zeta$ or that $\xi$ and $\zeta$ are independent (we denote by $i D$ the functionally dependent case, and $i I$ the latter case).

Proposition 3 Let $\tilde{a}$ and $\tilde{b}$ be two continuous fuzzy intervals with underlying continuous random variables $\xi, \zeta$.

1. $\mu_{1}(\tilde{a}, \tilde{b})=1-\mu_{4}(\tilde{b}, \tilde{a})$

2. $\mu_{1}(\tilde{a}, \tilde{b}) \leq \mu_{i}(\tilde{a}, \tilde{b}) \leq \mu_{4}(\tilde{a}, \tilde{b}), i \in\{2,3\}$

3. $\mu_{1}(\tilde{a}, \tilde{b})>0 \Rightarrow \mu_{4}(\tilde{a}, \tilde{b})=1$

4. $\quad \mu_{2}(\tilde{a}, \tilde{b})=1-\mu_{2}(\tilde{b}, \tilde{a})$ if $P\left(\underline{a}^{\xi}=\underline{b}^{\zeta}\right)=0$.

5. $\mu_{3}(\tilde{a}, \tilde{b})=1-\mu_{3}(\tilde{b}, \tilde{a})$ if $P\left(\bar{a}^{\xi}=\bar{b}^{\zeta}\right)=0$.

Proof The first item is obvious if one notices that $P\left(\underline{a}^{\xi}=\bar{b}^{\zeta}\right)=0$, due to continuity assumptions. The second item follows from the relative strength of the relations $>{ }_{i}$. For the third, notice that $\mu_{1}(\tilde{a}, \tilde{b})>0$ means that $\underline{a}^{\alpha}>\bar{b}^{\beta}$ for some $\xi=\alpha, \zeta=\beta>0$. It means that $\bar{a}^{1}>\underline{b}^{1}$, hence $\bar{a}^{\xi}>\underline{b}^{\zeta}, \forall \xi, \zeta>0$. Finally, the two last properties are due to the fact that $P(a>b)+P(b>a)+P(a=b)=1$.

No assumption of independence between $\xi$ and $\zeta$ is needed to obtain these obvious results, a consequence of which is:

Corollary 1 (Chanas and Zielinski 1999) Let ã and $\tilde{b}$ be two continuous fuzzy intervals with underlying continuous random variables $\xi, \zeta$. Then $\mu_{1}(\tilde{a}, \tilde{b})>0 \Longrightarrow \underline{a}^{1}>\bar{b}^{1}$ and $\mu_{4}(\tilde{a}, \tilde{b})<1 \Longleftrightarrow \bar{a}^{1}<\underline{b}^{1}$ (or equivalently $\mu_{4}(\tilde{a}, \tilde{b})=1 \Longleftrightarrow \bar{a}^{1} \geq \underline{b}^{1}$ ).

It is interesting to notice that counterparts to properties 4 and 5 in Proposition 3 hold for possibilistic indices $\operatorname{pos}_{3}$ and $n e c_{2}$, as previously recalled: such comparison indices define reciprocal fuzzy relations. 


\subsubsection{The case of $L-R$ fuzzy intervals with dependence assumptions}

Suppose that the fuzzy intervals have the same shape, up to a homothety, i.e are of the $L-R$ type, that is, $\tilde{a}=\left(\underline{a}, \bar{a}, \delta^{a}, \gamma^{a}\right) \in \mathcal{F}_{L R}(\mathbb{R})$ and $\tilde{b}=\left(\underline{b}, \bar{b}, \delta^{b}, \gamma^{b}\right) \in \mathcal{F}_{L R}(\mathbb{R})$. In the whole section $L$ and $R$ are continuous and strictly decreasing. The above fuzzy relations with the random interval approach can be expressed in the functionally dependent case $(\xi=\zeta)$ by:

1. $\mu_{1 D}(\tilde{a}, \tilde{b})=P\left(\underline{a}-L^{-1}(\xi) \delta^{a}>\bar{b}+R^{-1}(\xi) \gamma^{b}\right)$

2. $\mu_{2 D}(\tilde{a}, \tilde{b})=P\left(\bar{a}-L^{-1}(\xi) \delta^{a}>b-L^{-1}(\xi) \delta^{b}\right)$

3. $\mu_{3 D}(\tilde{a}, \tilde{b})=P\left(\bar{a}+R^{-1}(\xi) \gamma^{a}>\bar{b}+R^{-1}(\xi) \gamma^{b}\right)$

4. $\mu_{4 D}(\tilde{a}, \tilde{b})=P\left(\bar{a}+R^{-1}(\xi) \gamma^{a}>\underline{b}-L^{-1}(\xi) \delta^{b}\right)$.

The letter $\mathrm{D}$ stands for this dependence assumption. Chanas et al. consider two additional cases where $\xi$ and $\zeta$ are independent random variables with the uniform distribution on interval $[0,1]$ :

1. $\mu_{1 I}(\tilde{a}, \tilde{b})=P\left(\underline{a}-L^{-1}(\xi) \delta^{a}>\bar{b}+R^{-1}(\zeta) \gamma^{b}\right)$

2. $\mu_{4 I}(\tilde{a}, \tilde{b})=P\left(\overline{\bar{a}}+R^{-1}(\zeta) \gamma^{a}>\underline{b}-L^{-1}(\xi) \delta^{a}\right)$.

The $L-R$ setting allows for explicit calculations. Namely, since $P$ is a uniform distribution, then if $L$ and $R$ are strictly decreasing and continuous, one can easily see that

- If $\bar{b}<\underline{a}$ and $\underline{a}-\delta^{a}<\bar{b}+\gamma^{b}$ then there is a single $\xi=\alpha_{1}$ such that $\underline{a}-L^{-1}(\xi) \delta^{a}=$ $\bar{b}+R^{-1}(\xi) \gamma^{b}$. It is such that $0<\alpha_{1}<1$. If $L=R, \alpha_{1}=L\left(\frac{a-\bar{b}}{\delta^{a}+\gamma^{b}}\right)$ hence $\mu_{1 D}(\tilde{a}, \tilde{b})=1-\alpha_{1}=1-L\left(\frac{a-\bar{b}}{\delta^{a}+\gamma^{b}}\right)=\operatorname{nec}(\tilde{a}>\tilde{b})$. Otherwise, if $\bar{b} \geq \underline{a}$ then $\mu_{1 D}(\tilde{a}, \tilde{b})=0$ and if $\underline{a}-\delta^{a} \geq \underline{b}+\delta^{b}$, then $\mu_{1 D}(\tilde{a}, \tilde{b})=1$.

- Since $\mu_{1 D}(\tilde{a}, \tilde{b})=1-\mu_{4 D}(\tilde{b}, \tilde{a})$ then we have $\mu_{4 D}(\tilde{a}, \tilde{b})=L\left(\frac{\underline{b}-\bar{a}}{\gamma^{a}+\delta^{b}}\right)=$ $\operatorname{pos}(\tilde{a} \leq \tilde{b})$, when $\bar{a}<\underline{b}$ and $\bar{a}+\gamma^{a}>\underline{b}-\delta^{a}$.

So in case 1 and 4 under comonotonic dependence assumption, possibilistic indices 1 and 4 coincide with the random interval ones. There is a condition that is assumed in the above development: $\mu_{1 D}(\tilde{a}, \tilde{b})=\operatorname{nec}(\tilde{a}>\tilde{b})$ is true if the increasing part of the membership function of $\tilde{a}$ intersects the decreasing part of the membership function of $\tilde{b}$ only once. Namely, the set $I=\left\{\xi, \underline{a}-L^{-1}(\xi) \delta^{a}>\bar{b}+R^{-1}(\xi) \gamma^{b}\right\}$ is of the form $\left(\alpha_{1}, 1\right]$, whose Lebesgue measure is $1-\alpha_{1}$. If this condition does not hold the set $I$ will not be of the form $\left(\alpha_{1}, 1\right]$, and its Lebesgue measure will differ from the degree of necessity of dominance. Similar considerations can be formulated for $\mu_{4 D}(\tilde{a}, \tilde{b})=\operatorname{pos}(\tilde{a} \geq \tilde{b})$.

Likewise in case $2 D$ and $3 D$ :

- If $\underline{a} \geq \underline{b}$ but $\underline{a}-\delta^{a}<\underline{b}-\delta^{b}$ one can solve the equation $\underline{a}-L^{-1}(\xi) \delta^{a}=$ $\underline{b}-L^{-1}(\xi) \delta^{b}$. The single solution of which is $\alpha_{2}=L\left(\frac{\underline{a}-\underline{b}}{\delta^{a}-\delta^{b}}\right)$. Then $\mu_{2 D}(\tilde{a}, \tilde{b})=$ $1-\alpha_{2}=1-L\left(\frac{\underline{a}-\underline{b}}{\delta^{a}-\delta^{b}}\right)$

- In the same way, if $\bar{a} \leq \bar{b}$ but $\bar{a}+\gamma^{a}>\bar{b}+\gamma^{b}$, one can solve the equation $\bar{a}+R^{-1}(\xi) \gamma^{a}=\bar{b}+R^{-1}(\xi) \gamma^{b}$, the single solution of which is $\alpha_{3}=R\left(\frac{\bar{b}-\bar{a}}{\gamma^{a}-\gamma^{b}}\right)$; so $\mu_{3 D}(\tilde{a}, \tilde{b})=\alpha_{3}=R\left(\frac{\bar{b}-\bar{a}}{\gamma^{a}-\gamma^{b}}\right)$. 
Consequently the membership functions $\mu_{i D}$ verify the following properties:

$$
\begin{aligned}
& \mu_{2 D}(\tilde{a}, \tilde{b})= \begin{cases}L\left(\frac{a}{\delta^{a}-\underline{b}}\right) & \text { for } \underline{a}-\delta^{a}>\underline{b}-\delta^{b} \text { and } \underline{a} \leq \underline{b} \\
1 & \text { for } \underline{a}-\delta^{a}>\underline{b}-\delta^{b} \text { and } \underline{a}>\underline{b} \\
1-L\left(\frac{\underline{a}-\underline{b}}{\delta^{a}-\delta^{b}}\right) & \text { for } \underline{a}-\delta^{a}<\underline{b}-\delta^{b} \text { and } \underline{a} \geq \underline{b} \\
0 & \text { for } \underline{a}-\delta^{a} \leq \underline{b}-\delta^{b} \text { and } \underline{a}<\underline{b}\end{cases}
\end{aligned}
$$

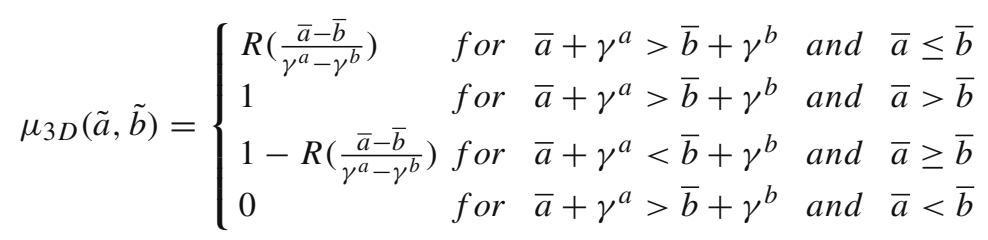

We can specialize the last items of Proposition 3:

Corollary 2 (Chanas and Zielinski 1999) Let $\tilde{a}=\left(\underline{a}, \bar{a}, \delta^{a}, \gamma^{a}\right) \in \mathcal{F}_{L R}(\mathbb{R})$ and $\tilde{b}=\left(\underline{b}, \bar{b}, \delta^{b}, \gamma^{b}\right) \in \mathcal{F}_{L R}(\mathbb{R})$ be two fuzzy intervals of type $L-R$

- If $\delta^{a} \neq \delta^{b}$ or $\underline{a} \neq \underline{b}$ then $\mu_{2 D}(\tilde{a}, \tilde{b})+\mu_{2 D}(\tilde{b}, \tilde{a})=1$.

- If $\gamma^{a} \neq \gamma^{b}$ or $\bar{a} \neq \bar{b}$ then $\mu_{3 D}(\tilde{a}, \tilde{b})+\mu_{3 D}(\tilde{b}, \tilde{a})=1$.

Indeed if $\delta^{a}=\delta^{b}$ and $\underline{a}=\underline{b}$, the left hand side of the fuzzy numbers are equal and $\mu_{2 D}(\tilde{a}, \tilde{b})=\mu_{2 D}(\tilde{b}, \tilde{a})=0$ while the probability of equality is one. Likewise if $\gamma^{a}=\gamma^{b}$ and, $\bar{a}=\bar{b}$ for $\mu_{3 D}(\tilde{a}, \tilde{b})$ on the right hand side of the fuzzy numbers.

Proposition 4 Let $\tilde{a}=\left(\underline{a}, \bar{a}, \delta^{a}, \gamma^{a}\right) \in \mathcal{F}_{L R}(\mathbb{R})$ and $\tilde{b}=\left(\underline{b}, \bar{b}, \delta^{b}, \gamma^{b}\right) \in \mathcal{F}_{L R}(\mathbb{R})$ be two fuzzy intervals of type $L-R$ with $L$ and $R$ strictly decreasing and continuous. Then, $\forall \beta \in[0,1], \beta \neq 0,1$ :

- if $L=R$ and $\bar{b}<\underline{a}$ and $\underline{a}-\delta^{a}<\bar{b}+\gamma^{b}$ then $\mu_{1 D}(\tilde{a}, \tilde{b}) \geq \beta$ if and only if $\underline{a}-\bar{b}-L^{-1}(1-\beta)\left(\delta^{a}+\bar{\gamma}^{b}\right) \geq 0$

- If $\underline{a}>\underline{b}$ but $\underline{a}-\delta^{a}<\underline{b}+\delta^{b}$ then $\mu_{2 D}(\tilde{a}, \tilde{b}) \geq \beta$ if and only if $\underline{a}-\underline{b}-L^{-1}(1-$ $\beta)\left(\delta^{a}-\delta^{b}\right) \geq 0$;

- if $\bar{a}<\bar{b}$ but $\bar{a}-\gamma^{a}>\bar{b}+\gamma^{b}$ then $\mu_{3 D}(\tilde{a}, \tilde{b}) \geq \beta$ if and only if $\bar{a}-\bar{b}+$ $R^{-1}(\beta)\left(\gamma^{a}-\gamma^{b}\right) \geq 0$

- if $L=R$ and if $\underline{b}>\bar{a}$ and $\bar{a}+\gamma^{a}>\underline{b}+\delta^{b}$ then $\mu_{4 D}(\tilde{a}, \tilde{b}) \geq \beta$ if and only if $\underline{b}-\bar{a}-L^{-1}(\beta)\left(\gamma^{a}+\delta^{b}\right) \leq 0$

Proof (For instance)

- We have $\forall \beta \in(0,1]: \mu_{2 D}(\tilde{a}, \tilde{b}) \geq \beta \Longleftrightarrow 1-L\left(\frac{a-b}{\delta^{a}-\delta^{b}}\right) \geq \beta \Longleftrightarrow$ $L\left(\frac{a-b}{\delta^{a}-\delta^{b}}\right) \leq 1-\beta$ and since $L$ is strictly decreasing, thus $L^{-1}$ is strictly decreasing, then we obtain $\frac{a-b}{\delta^{a}-\delta^{b}} \geq L^{-1}(1-\beta)$, then $\underline{a}-\underline{b}-L^{-1}(1-\beta)\left(\delta^{a}-\delta^{b}\right) \geq 0$.

- In the same way $\mu_{3 D}(\tilde{a}, \tilde{b}) \geq \beta \Longleftrightarrow R\left(\frac{\bar{b}-\bar{a}}{\gamma^{a}-\gamma^{b}}\right) \geq \beta$ and since $R$ is strictly decreasing, thus $R^{-1}$ is strictly decreasing, then we obtain $\frac{\bar{b}-\bar{a}}{\gamma^{a}-\gamma^{b}} \leq R^{-1}(\beta)$, then $\bar{a}-\bar{b}+R^{-1}(\beta)\left(\gamma^{a}-\gamma^{b}\right) \geq 0$. 
Note that for $\beta=1, \mu_{1 D}(\tilde{a}, \tilde{b})=1$ if and only if $\underline{a}-\delta^{a} \geq \bar{b}+\gamma^{b}$, and $\mu_{4 D}(\tilde{a}, \tilde{b})=1$ if and only if $\bar{a} \geq \underline{b}$. For the two other indices, Chanas and Zielinski (1999) mention the following consequence:

Corollary 3 Let $\tilde{a}=\left(\underline{a}, \bar{a}, \delta^{a}, \gamma^{a}\right) \in \mathcal{F}_{L R}(\mathbb{R})$ and $\tilde{b}=\left(\underline{b}, \bar{b}, \delta^{b}, \gamma^{b}\right) \in \mathcal{F}_{L R}(\mathbb{R})$ be two fuzzy intervals of type $L-R$ with $L$ and $R$ strictly decreasing and continuous. We have then:

- $\mu_{2 D}(\tilde{a}, \tilde{b}) \geq \frac{1}{2} \Longleftrightarrow \underline{a}-\underline{b}-L^{-1}\left(\frac{1}{2}\right)\left(\delta^{a}-\delta^{b}\right) \geq 0$;

- $\mu_{3 D}(\tilde{a}, \tilde{b}) \geq \frac{1}{2} \Longleftrightarrow \bar{a}-\bar{b}+R^{-1}\left(\frac{1}{2}\right)\left(\gamma^{a}-\gamma^{b}\right) \geq 0$;

This result uses the value $1 / 2$ as a threshold due the fact that $\mu_{2 D}, \mu_{D 3}$ are reciprocal relations (see Corollary 2). So only if $\mu_{i D}(\tilde{a}, \tilde{b}) \geq \alpha>\frac{1}{2}, i=2,3$ does it mean that $\tilde{a}$ dominates $\tilde{b}$.

The other assumption used by Chanas et al. is that the cuts of $\widetilde{a}$ and $\tilde{b}$ are induced by two independent random variables $\xi$ and $\zeta$ on the unit interval. It is the case of two fuzzy intervals supplied by independent sources. One then speaks of fuzzy intervals with independent confidence levels. The explicit calculation of indices can also be carried out. For instance, if $\bar{b}<\underline{a}$ and $\underline{a}-\delta^{a}<\bar{b}+\gamma^{b}$ then $\mu_{1 I}(\tilde{a}, \tilde{b})$ is the surface above the line defined by $\underline{a}-\delta^{a} L^{-1}(\xi)=\bar{b}+\gamma^{b} R^{-1}(\zeta)$ in the unit square. Namely we must have $\underline{a}-\delta^{a} L^{-1}(\bar{\xi})<\bar{b}+\gamma^{b} R^{-1}(\zeta)$ to have overlapping cuts. Hence

$$
\mu_{1 I}(\tilde{a}, \tilde{b})=1-\int_{0}^{1} R\left(\min \left(1, \max \left(0, \frac{a-\bar{b}-\delta^{a} L^{-1}(\xi)}{\gamma^{b}}\right)\right)\right) d \xi
$$

When $L$ and $R$ are linear, it is possible to compute an explicit value analytically. Moreover, the two events $\left\{\xi: \underline{a}-L^{-1}(\xi) \delta^{a}>\underline{b}-L^{-1}(\xi) \delta^{b}\right\}$ and $\left\{\zeta: \bar{a}+R^{-1}(\zeta) \gamma^{a}>\right.$ $\left.\bar{b}+R^{-1}(\zeta) \gamma^{b}\right\}$ being independent, a valued extension of the canonical interval-lattice order relation $\succ$ can be defined as follows:

$$
\mu_{\succ}^{I}(\tilde{a}, \tilde{b})=\mu_{2 D}(\tilde{a}, \tilde{b}) \cdot \mu_{3 D}(\tilde{a}, \tilde{b}) .
$$

\subsubsection{Ordering fuzzy quantities via scalar representatives}

Another approach to compare fuzzy intervals consists in choosing real numbers that may represent them, and rank the fuzzy intervals accordingly. This process is often called defuzzification, even if defuzzifying a fuzzy interval should yield an interval (Ogura et al. 2001). The latter view is the natural one if we admit that fuzzy intervals represent incomplete information. Then the selection process is as follows:

- Compute an interval $I(\tilde{a})$ from a fuzzy interval $\tilde{a}$. In agreement with the random interval view, it is natural to define this interval as the interval average (Dubois and Prade 1987b; Ogura et al. 2001): I $(\tilde{a})=\left[\int_{0}^{1} \underline{a}^{\alpha} d \alpha, \int_{0}^{1} \bar{a}^{\alpha} d \alpha\right]$.

- Select an element in this interval: It depends on the attitude of the decisionmaker (that is, optimistic or pessimistic). This element can be of the form $\sigma(\tilde{a})=$ $\lambda \inf I(\tilde{a})+(1-\lambda) \sup I(\tilde{a})$. This is the well-known Hurwicz criterion. 
This approach can be found in the literature in various forms. The older proposal of this kind is due to Yager $(1978,1980,1993)$ where $\lambda=1 / 2$, i.e. the midpoint of the mean interval is chosen. Fortemps and Roubens method (Fortemps and Roubens 1996) comes down to ranking fuzzy intervals according to the same scalar substitute as Yager. The most general case including the decision-maker attitude via the choice of $\lambda$ corresponds to the approach proposed independently by de Campos and Gonzalez Munoz (1989) and Liou and Wang (1992). The linearity of the indices stemming from the above approach is well-known:

- $\sigma(\tilde{a}+\tilde{b})=\sigma(\tilde{a})+\sigma(\tilde{a})$.

- $\sigma(r \tilde{a})=r \sigma(\tilde{a}), r$ is a real number.

However, turning a fuzzy interval into a single number can be debatable in some situations as it gets rid of the information concerning uncertainty.

\section{Various formulations of fuzzy chance constraints}

We consider a set of linear constraints bearing on $n$ variables represented by a matrix $A(m \times n)$ and a vector $b(m \times 1)$ whose components are, respectively $a_{i j}$ and $b_{i}$. The constraints of the fuzzy stochastic problem $\left(P_{F S}\right)$ can be written as follows:

$$
\begin{array}{r}
\tilde{b}_{i}(\omega) \geq \sum_{j=1}^{n} \tilde{a}_{i j}(\omega) \odot x_{j}, \\
x_{j} \geq 0, j=1, \ldots, n
\end{array}
$$

$\sum_{j=1}^{n}$ and $\odot$ represent, for given $\omega \in \Omega$, the addition of fuzzy intervals of type $L-R$ and their multiplication by a real number, respectively (see Appendix A). Note that here we assume that $\omega$ is a scenario where the coefficients of matrix $A$ and $b$ are simultaneously determined, but ill-observed. The order relation $\geq$ must then be given a meaning. When $\tilde{a}_{i j}(\omega)=\left(\underline{a}_{i j}(\omega), \bar{a}_{i j}(\omega), \delta_{i j}^{a}, \gamma_{i j}^{a}\right)$ and $\tilde{b}_{i}=\left(\underline{b}_{i}(\omega), \bar{b}_{i}(\omega), \delta_{i}^{b}, \gamma_{i}^{b}\right)$ are fuzzy random variables of type $L-R$, then

$$
\sum_{j=1}^{n} \tilde{a}_{i j}(\omega) \odot x_{j}=\left(\sum_{j=1}^{n} \underline{a}_{i j}(\omega) x_{j}, \sum_{j=1}^{n} \bar{a}_{i j}(\omega) x_{j}, \sum_{j=1}^{n} \delta_{i j}^{a} x_{j}, \sum_{j=1}^{n} \gamma_{i j}^{a} x_{j}\right)
$$

is also of type $L-R$. In what follows, $A$ (resp. $b$ ) is deterministic, fuzzy, stochastic or fuzzy stochastic according to whether the coefficients $a_{i j}$ (resp. $b_{i}$ ) are, respectively deterministic, fuzzy intervals, random variables or fuzzy random variables.

Deterministic counterparts of constraints in the chance-constrained fuzzy programming problem will then take the following form:

$$
P\left(\rho\left(\tilde{b}_{i}(\omega), \sum_{j=1}^{n} \tilde{a}_{i j}(\omega) \odot x_{j}\right) \geq \beta_{i}\right) \geq p_{i}, i=1, \ldots, m
$$


where $\rho(\tilde{a}, \tilde{b})$ evaluates the degree of confidence to which the coefficient restricted by $\tilde{a}$ is greater than the coefficient restricted by $\tilde{b}$.

In order to convert fuzzy stochastic constraints of $\left(P_{F S}\right)$, into deterministic ones, we consider four versions, according to the choice of $\rho$. We can use the degrees of possibility, of necessity of preference, or the Chanas et al. indices of stochastic preference for random intervals. We can also let $\rho(\tilde{a}, \tilde{b})$ encode the comparison of scalar substitutes of fuzzy intervals. In the following we use membership functions that satisfy assumptions needed for ensuring the application of the results in previous sections.

\subsection{Combining probability and possibility}

A fuzzy-stochastic constraint in problem $\left(P_{F S}\right)$ can be expressed using possibility of dominance as:

$$
\left(P_{p}\right):\left\{\begin{array}{l}
\max \phi(x) \\
P\left(\omega: \operatorname{pos}\left(\sum_{j=1}^{n} \tilde{a}_{i j}(\omega) \odot x_{j} \leq \tilde{b}_{i}(\omega)\right) \geq \beta_{i}\right) \geq p_{i}, i=1, \ldots, m \\
x_{j} \geq 0, j=1, \ldots, n
\end{array}\right.
$$

where $P$ and pos denote, respectively probability and possibility. This formulation, used for instance by Katagiri et al. (2004) is very weak since even if $\beta_{i}=p_{i}=1$ there is no certainty about the satisfaction of this constraint.

A feasible solution $x^{0}=\left(x_{1}^{0}, x_{2}^{0}, \ldots, x_{n}^{0}\right) \geq 0$ to problem $\left(P_{p}\right)$ is called pro-pos feasible.

Proposition 5 The set of pro-pos feasible solutions to problem $\left(P_{p}\right)$, denoted by $\mathcal{X}_{p}^{i}\left(p_{i}, \beta_{i}\right)$ can be written as follows:

1. If $\tilde{a}_{i j}(\omega)$ and $\tilde{b}_{i}(\omega)$ are fuzzy random variables then:

$\mathcal{X}_{p}^{i}\left(p_{i}, \beta_{i}\right)=\left\{x \geq 0: P\left(\omega: \sum_{j=1}^{n} \underline{a}_{i j}^{\beta_{i}}(\omega) x_{j} \leq \bar{b}_{i}^{\beta_{i}}(\omega)\right) \geq p_{i}\right\}, i=1, \ldots, m$ where $\underline{a}_{i j}^{\beta_{i}}(\omega)$ and $\bar{b}_{i}^{\beta_{i}}(\omega)$ are, respectively lower and upper bounds of the corresponding $\tilde{a}_{i j}^{\beta_{i}}(\omega)$ and $\tilde{b}_{i}^{\beta_{i}}(\omega)$.

2. If $\tilde{a}_{i j}(\omega)=\left(\underline{a}_{i j}(\omega), \bar{a}_{i j}(\omega), \delta_{i j}^{a}, \gamma_{i j}^{a}\right)$ and $\tilde{b}_{i}(\omega)=\left(\underline{b}_{i}(\omega), \bar{b}_{i}(\omega), \delta_{i}^{b}, \gamma_{i}^{b}\right)$ are fuzzy random variables of type $L-R$, then:

$\mathcal{X}_{p}^{i}\left(p_{i}, \beta_{i}\right)=\left\{x \geq 0: P\left(\omega: \sum_{j=1}^{n}\left(\underline{a}_{i j}(\omega)-L^{-1}\left(\beta_{i}\right) \delta_{i j}^{a}\right) x_{j} \leq \bar{b}_{i}(\omega)+\right.\right.$ $\left.\left.R^{-1}\left(\beta_{i}\right) \gamma_{i}^{b}\right) \geq p_{i}\right\}, i=1, \ldots, m$.

The proof is obvious, it is enough to use properties of possibility measures given in Dubois (1987) and recalled in Sect. 3.3.1. The brittle nature of the solutions to this constraint is clear as it means that it is satisfied as soon its least demanding crisp counterpart is satisfied; but there is no guarantee that it will be the case in practice. 


\subsection{Combining probability and necessity}

A fuzzy-stochastic constraint in problem $\left(P_{F S}\right)$ can be expressed using necessity of dominance as:

$$
\left(P_{n}\right):\left\{\begin{array}{l}
\max \phi(x) \\
P\left\{\omega: \operatorname{nec}\left(\sum_{j=1}^{n} \tilde{a}_{i j}(\omega) \odot x_{j} \leq \tilde{b}_{i}(\omega)\right) \geq \beta_{i}\right\} \geq p_{i}, i=1, \ldots, m \\
x_{j} \geq 0, j=1, \ldots, n
\end{array}\right.
$$

where $P$ and nec denote, respectively probability and necessity. This deterministic expression of the constraint ensures its robustness to level $\beta_{i}$ together with its frequent satisfaction according to level $p_{i}$. A feasible solution $x^{0}=\left(x_{1}^{0}, x_{2}^{0}, \ldots, x_{n}^{0}\right) \geq 0$ to problem $\left(P_{n}\right)$ is called pro-nec feasible.

Proposition 6 The set of pro-nec feasible solutions to problem $\left(P_{n}\right)$, denoted by $\mathcal{X}_{n}^{i}\left(p_{i}, \beta_{i}\right)$, can be written as follows:

1. If $\tilde{a}_{i j}(\omega)$ and $\tilde{b}_{i}(\omega)$ are fuzzy random variables then:

$\mathcal{X}_{n}^{i}\left(p_{i}, \beta_{i}\right)=\left\{x \geq 0: P\left(\omega: \sum_{j=1}^{n} \bar{a}_{i j}^{1-\beta_{i}}(\omega) x_{j} \leq \underline{b}_{i}^{1-\beta_{i}}(\omega)\right) \geq p_{i}\right\}, i=$

where $\bar{a}_{i j}^{1-\beta_{i}}(\omega)$ and $\underline{b}_{i}^{1-\beta_{i}}(\omega)$ are, respectively lower and upper bounds of the corresponding $\tilde{a}_{i j}^{1-\beta_{i}}(\omega)$ and $\tilde{b}_{i}^{1-\beta_{i}}(\omega)$.

2. If $\tilde{a}_{i j}(\omega)=\left(\underline{a}_{i j}(\omega), \bar{a}_{i j}(\omega), \delta_{i j}^{a}, \gamma_{i j}^{a}\right)$ and $\tilde{b}_{i}(\omega)=\left(\underline{b}_{i}(\omega), \bar{b}_{i}(\omega), \delta_{i}^{b}, \gamma_{i}^{b}\right)$ are fuzzy random variables of type $L-R$, then:

$\mathcal{X}_{n}^{i}\left(p_{i}, \beta_{i}\right)=\left\{x \geq 0: P\left(\omega: \sum_{j=1}^{n}\left(\bar{a}_{i j}(\omega)+R^{-1}\left(1-\beta_{i}\right) \gamma_{i j}^{a}\right) x_{j} \leq \underline{b}_{i}(\omega)-\right.\right.$ $\left.\left.L^{-1}\left(1-\beta_{i}\right) \delta_{i}^{b}\right) \geq p_{i}\right\}, i=1, \ldots, m$.

The proof is obvious (it is enough to use properties of necessity given in Dubois (1987) and recalled in Sect. 3.3.1). The robust nature of the solutions to this constraint is clear as it means that its solution is probably feasible, whatever the actual value of the coefficients in the $1-\beta_{i}$ cuts of the fuzzy sets $\tilde{a}_{i j}(\omega)$ and $\tilde{b}_{i}(\omega)$, when $\omega$ is fixed.

Note that the same type of reasoning can be followed for handling indices nec 2 and pos $_{3}$ when comparing fuzzy numbers. However, the meaning of solutions will again differ. Indeed using the latter indices comes down to assuming that all coefficients take pessimistic or optimistic values simultaneously. Propositions similar to the above ones can be written using Proposition 2.

4.3 Combining probability and scalar indices for ordering of fuzzy quantities

Due to the assumed linearity of the defuzifying operation $\sigma$, the problem then writes:

$$
\left(P_{\sigma}\right)\left\{\begin{array}{l}
\max \phi(x) \\
P\left\{\omega: \sum_{j=1}^{n} \sigma\left(\tilde{a}_{i j}(\omega)\right) x_{j} \leq \sigma\left(\tilde{b}_{i}(\omega)\right)\right\} \geq p_{i}, i=1, \ldots, m \\
x_{j} \geq 0, j=1, \ldots, n
\end{array}\right.
$$


where $P$ denote probability and $\sigma(\tilde{a})$ is a scalar substitute of $\tilde{a}$. It is obvious that $\sigma\left(\tilde{a}_{i j}(\omega)\right)$ and $\sigma\left(\tilde{b}_{i}(\omega)\right)$ are real random variables. The problem then comes down to standard chance-constrained programming. A feasible solution $x^{0}=$ $\left(x_{1}^{0}, x_{2}^{0}, \ldots, x_{n}^{0}\right) \geq 0$ to problem $\left(P_{\sigma}\right)$ is called pro- $\sigma$ feasible. The set of pro $-\sigma$ feasible solutions to problem $\left(P_{\sigma}\right)$ is denoted by $\mathcal{X}_{\sigma}^{i}\left(p_{i}\right)$. This drastic simplification comes along with difficulties to interpret the solution to such a formulation. Indeed if $\sigma$ is given by a defuzzification scheme that has no clear rationale, then the obtained solution cannot be interpreted. If $\sigma$ computes an Hurwiczlike substitute depending on a coefficient of pessimism $\lambda$, it is easier to interpret, but it highlights the fact that replacing a fuzzy interval by a crisp number is the responsibility of the decision-maker, and has no objectively defendable justification.

4.4 Combining chance-constrained programming and random interval comparison

Now we assume that in each scenario $\omega$, there is a random process that governs the definition of interval coefficients in problem $\left(P_{F S}\right)$, which now takes the form:

$$
\left(P_{\mu_{k}}\right):\left\{\begin{array}{l}
\max \phi(x) \\
P\left\{\omega: \mu_{k}\left(\tilde{b}_{i}(\omega), \sum_{j=1}^{n} \tilde{a}_{i j}(\omega) \odot x_{j}\right) \geq \beta_{i}\right\} \geq p_{i}, i=1, \ldots, m ; k \\
=1 D, 2 D, 3 D, 4 D, 1 I, 4 I \\
x_{j} \geq 0, j=1, \ldots, n
\end{array}\right.
$$

where the indices $\mu_{k}$ are stochastic extensions of some interval-related ordering. A feasible solution $x^{0}=\left(x_{1}^{0}, x_{2}^{0}, \ldots, x_{n}^{0}\right) \geq 0$ to problem $\left(P_{\mu_{k}}\right)$ is called pro$\mu_{k}$ feasible. The set of pro- $\mu_{k}$ feasible solutions to problem $\left(P_{\mu_{k}}\right)$ is denoted by $\mathcal{X}_{\mu_{k}}^{i}\left(p_{i}, \beta_{i}\right)$. We restrict to the case of functionally related random variables underlying the fuzzy coefficients, and distinguish the case of fuzzy ordering relations generalizing interval orderings (case $k=1 D, 4 D$ ) from the case when they are reciprocal $(k=2 D, 3 D)$. In the latter case, we need $\beta_{i} \geq \frac{1}{2}$ to make sense of the inequality.

Cases 2D and 3D Based on the definition of $\mathcal{X}_{\mu_{k}}\left(p_{i}, \beta_{i}\right)$ and Corollary 1, we will rewrite the feasible sets $\mathcal{X}_{\mu_{k}}^{i}\left(p_{i}, \beta_{i}\right), k=2 D, 3 D, \beta_{i} \geq \frac{1}{2}$ as follows:

Proposition 7 Let $\tilde{b}_{i}(\omega)=\left(\underline{b}_{i}(\omega), \bar{b}_{i}(\omega), \delta_{i}^{b}, \gamma_{i}^{b}\right)$ and $\tilde{a}_{i j}(\omega)=\left(\underline{a}_{i j}(\omega), \bar{a}_{i j}(\omega)\right.$, $\left.\delta_{i j}^{a}, \gamma_{i j}^{a}\right)$ be fuzzy random variables of the type $L-R$. Under assumptions of Corollary 2 and $\beta_{i} \geq \frac{1}{2}$, we have:

- $\mathcal{X}_{\mu_{2 D}}^{i}\left(p_{i}, \beta_{i}\right)=\left\{x \geq 0: P\left(\omega: \sum_{j=1}^{n}\left(\underline{a}_{i j}(\omega)-L^{-1}\left(1-\beta_{i}\right) \delta_{i j}^{a}\right) x_{j} \leq \underline{b}_{i}(\omega)-\right.\right.$ $\left.\left.L^{-1}\left(1-\beta_{i}\right) \delta_{i}^{b}\right) \geq p_{i}\right\}$.

- $\mathcal{X}_{\mu_{3 D}}^{i}\left(p_{i}, \beta_{i}\right)=\left\{x \geq 0: P\left(\omega: \sum_{j=1}^{n}\left(\bar{a}_{i j}(\omega)+R^{-1}\left(\beta_{i}\right) \gamma_{i j}^{a}\right) x_{j} \leq \bar{b}_{i}(\omega)+\right.\right.$ $\left.\left.R^{-1}\left(\beta_{i}\right) \gamma_{i}^{b}\right) \geq p_{i}\right\}$. 
Proof We do not need $\beta_{i} \geq \frac{1}{2}$ in the proof. By definition for $i=1, \ldots, m$ :

$$
\begin{aligned}
& \mathcal{X}_{\mu_{2 D}}^{i}\left(p_{i}, \beta_{i}\right)=\left\{x=\left(x_{1}, x_{2}, \ldots, x_{n}\right) \geq 0: P\left(\omega: \mu_{2}\left(\tilde{b}_{i}(\omega), \sum_{j=1}^{n} \tilde{a}_{i j}(\omega) \odot x_{j}\right) \geq \beta_{i}\right) \geq p_{i}\right\} \\
& \mathcal{X}_{\mu_{3 D}}^{i}\left(p_{i}, \beta_{i}\right)=\left\{x=\left(x_{1}, x_{2}, \ldots, x_{n}\right) \geq 0: P\left(\omega: \mu_{3}\left(\tilde{b}_{i}(\omega), \sum_{j=1}^{n} \tilde{a}_{i j}(\omega) \odot x_{j}\right) \geq \beta_{i}\right) \geq p_{i}\right\}
\end{aligned}
$$

Then, from Proposition 4, we get, for $i=1, \ldots, m$ :

$$
\begin{aligned}
& \mu_{2 D}\left(\tilde{b}_{i}(\omega), \sum_{j=1}^{n} \tilde{a}_{i j}(\omega) \odot x_{j}\right) \geq \beta_{i} \Leftrightarrow \sum_{j=1}^{n}\left(\underline{a}_{i j}(\omega)-L^{-1}\left(1-\beta_{i}\right) \delta_{i j}^{a}\right) x_{j} \leq \underline{b}_{i}(\omega)-L^{-1}\left(1-\beta_{i}\right) \delta_{i}^{b} \\
& \mu_{3 D}\left(\tilde{b}_{i}(\omega), \sum_{j=1}^{n} \tilde{a}_{i j}(\omega) \odot x_{j}\right) \geq \beta_{i} \Leftrightarrow \sum_{j=1}^{n}\left(\bar{a}_{i j}(\omega)+R^{-1}\left(\beta_{i}\right) \gamma_{i j}^{a}\right) x_{j} \leq \bar{b}_{i}(\omega)+R^{-1}\left(\beta_{i}\right) \gamma_{i}^{b}
\end{aligned}
$$

It follows for $k=2 D, 3 D$ that:

$$
\begin{aligned}
& P\left(\mu_{2 D}\left(\tilde{b}_{i}, \sum_{j=1}^{n} \tilde{a}_{i j} \odot x_{j}\right) \geq \beta_{i}\right)=P\left(\left\{\omega: \sum_{j=1}^{n}\left(\underline{a}_{i j}(\omega)-L^{-1}\left(1-\beta_{i}\right) \delta_{i j}^{a}\right) x_{j} \leq \underline{b}_{i}(\omega)-L^{-1}\left(1-\beta_{i}\right) \delta_{i}^{b}\right\}\right) \\
& P\left(\mu_{3 D}\left(\tilde{b}_{i}, \sum_{j=1}^{n} \tilde{a}_{i j} \odot x_{j}\right) \geq \beta_{i}\right)=P\left(\omega: \sum_{j=1}^{n}\left(\bar{a}_{i j}(\omega)+R^{-1}\left(\beta_{i}\right) \gamma_{i j}^{a}\right) x_{j} \leq \bar{b}_{i}(\omega)+R^{-1}\left(\beta_{i}\right) \gamma_{i}^{b}\right)
\end{aligned}
$$

Cases 1D and 4D For a given $\omega \in \Omega$, from Proposition 4, we get:

$\forall 1 \geq \beta_{i}>0, \mu_{4} D\left(\tilde{b}_{i}(\omega), \sum_{j=1}^{n}\left(\tilde{a}_{i j}(\omega) \odot x_{j}\right) \geq \beta_{i} \Longleftrightarrow \sum_{j=1}^{n}\left(\underline{a}_{i j}(\omega)-\right.\right.$ $\left.L^{-1}\left(\beta_{i}\right) \delta_{i j}^{a}\right) x_{j} \leq \bar{b}_{i}(\omega)+R^{-1}\left(\beta_{i}\right) \gamma_{i}^{b}$.

Consequently $\left\{x \geq 0: P\left(\mu_{4} D\left(\tilde{b}_{i}(\omega), \sum_{j=1}^{n} \tilde{a}_{i j}(\omega) \odot x_{j}\right) \geq \beta_{i}\right) \geq p_{i}\right\}=\{x \geq 0$ : $\left.P\left(\omega: \sum_{j=1}^{n}\left(\underline{a}_{i j}(\omega)-L^{-1}\left(\beta_{i}\right) \delta_{i j}^{a}\right) x_{j} \leq \bar{b}_{i}(\omega)+R^{-1}\left(\beta_{i}\right) \gamma_{i}^{b}\right) \geq p_{i}\right\}$.

Likewise, for $k=1 D$, we get $\forall \beta_{i}<1, \mu_{1 D}\left(\tilde{b}_{i}(\omega), \sum_{j=1}^{n} \tilde{a}_{i j}(\omega) \odot x_{j}\right) \geq \beta_{i}$ in the form $\sum_{j=1}^{n}\left(\bar{a}_{i j}(\omega)+R^{-1}\left(1-\beta_{i}\right) \gamma_{i j}^{a}\right) x_{j} \leq \underline{b}_{i}(\omega)-L^{-1}\left(1-\beta_{i}\right) \gamma_{i}^{b}$. Consequently, $\left\{x \geq 0\right.$ : $P\left(\mu_{1 D}\left(\tilde{b}_{i}(\omega), \sum_{j=1}^{n}\left(\tilde{a}_{i j}(\omega) \odot x_{j}\right) \geq \beta_{i}\right) \geq p_{i}\right\}=\{x \geq 0$ : $\left.P\left(\omega: \sum_{j=1}^{n}\left(\bar{a}_{i j}(\omega)+R^{-1}\left(1-\beta_{i}\right) \gamma_{i j}^{a}\right) x_{j} \leq \underline{b}_{i}(\omega)-L^{-1}\left(1-\beta_{i}\right) \gamma_{i}^{b}.\right) \geq p_{i}\right\}$. Of course, if $\beta_{i}=1$, the feasible set reduces to $\left\{x \geq 0: P\left(\omega: \sum_{j=1}^{n}\left(\bar{a}_{i j}(\omega)+\gamma_{i j}^{a}\right) x_{j} \leq\right.\right.$ $\left.\left.\underline{b}_{i}(\omega)-\gamma_{i}^{b}.\right) \geq p_{i}\right\}$.

Remark We can easily see that: $\mathcal{X}_{p}^{i}\left(p_{i}, \beta_{i}\right)=\mathcal{X}_{\mu_{4 D}}^{i}\left(p_{i}, \beta_{i}\right)$ and $\mathcal{X}_{n}^{i}\left(p_{i}, \beta_{i}\right)=$ $\mathcal{X}_{\mu_{1 D}}^{i}\left(p_{i}, \beta_{i}\right)$.

Optimal solutions to such problems are defined as usual, since the various problems come down to checking the feasibility of deterministic constraints. 


\section{Convexity of feasible sets}

The feasible sets induced by fuzzy chance constraints can be convex, under some conditions as follows:

Theorem 1 If the requested probability levels are extreme, i.e. $p_{i}=0$ or $p_{i}=1$, then:

- $\mathcal{X}_{\sigma}^{i}\left(p_{i}\right)$ is convex.

- $\mathcal{X}_{\mu_{2 D}}^{i}\left(p_{i}, \beta_{i}\right)$ and $\mathcal{X}_{\mu_{3 D}}^{i}\left(p_{i}, \beta_{i}\right)$ are convex, for $\beta_{i} \geq \frac{1}{2}$.

- $\mathcal{X}_{p}^{i}\left(p_{i}, \beta_{i}\right)$ and $\mathcal{X}_{n}^{i}\left(p_{i}, \beta_{i}\right)$ are convex $\forall \beta_{i} \in(0,1]$.

Proof Obvious, it is enough to apply Theorem 5 of Appendix B.

Taking account of the conditions for the convexity of feasible sets resulting from the application of the chance-constrained programming method (Charnes and Cooper 1959) to linear stochastic programming and relying on results in Sect. 4, we distinguish the cases where $A$ is deterministic or fuzzy and those where $A$ is stochastic or fuzzy stochastic.

5.1 Subcases where $A$ is deterministic or fuzzy

We consider the sub-case where $A$ is fuzzy and $b$ is fuzzy stochastic and its components can be fuzzy random variables or $L-R$-fuzzy random variables. Based on the expression of sets of feasible solutions to chance constraints given in Sect. 4, and Theorem 5 of Appendix B, we establish the convexity of feasible sets:

Theorem 2 If the components of the matrix $A(m \times n)$ are fuzzy intervals $\tilde{a}_{i j}$ and those of the vector $b(m \times 1)$ are fuzzy random variables $\tilde{b}_{i}(\omega)$, then: $\forall \beta_{i} \in(0,1]$ and $\forall p_{i} \in[0,1]$, the feasible sets $\mathcal{X}_{p}^{i}\left(p_{i}, \beta_{i}\right), \mathcal{X}_{n}^{i}\left(p_{i}, \beta_{i}\right)$ and $\mathcal{X}_{\sigma}^{i}\left(p_{i}\right)$ are convex for all probability distributions of $\bar{b}_{i}^{\beta_{i}}, \underline{b}_{i}^{1-\beta_{i}}$ and defuzzifications $\sigma\left(\tilde{b}_{i}\right)$, respectively.

Proof Since $\tilde{a}_{i j}$ are fuzzy intervals, we replace $\underline{a}_{i j}^{\beta_{i}}(\omega)$ by $\underline{a}_{i j}^{\beta_{i}}$ in $\mathcal{X}_{p}^{i}\left(p_{i}, \beta_{i}\right)$, it follows that $\forall \beta_{i} \in(0,1]$ and $\forall p_{i} \in[0,1]$ :

$x \in \mathcal{X}_{p}^{i}\left(p_{i}, \beta_{i}\right) \Longleftrightarrow P\left(\omega: \sum_{j=1}^{n} \underline{a}_{i j}^{\beta_{i}} x_{j} \leq \bar{b}_{i}^{\beta_{i}}(\omega)\right) \geq p_{i} \Leftrightarrow$

$1-P\left(\omega: \sum_{j=1}^{n} \underline{a}_{i j}^{\beta_{i}}(\omega) x_{j} \geq \bar{b}_{i}^{\beta_{i}}(\omega)\right) \geq p_{i} \Leftrightarrow 1-\Psi_{\bar{b}_{i}^{\beta_{i}}}\left(\sum_{j=1}^{n} \underline{a}_{i j}^{\beta_{i}} x_{j}\right) \geq p_{i} \Leftrightarrow$ $\sum_{j=1}^{n} \underline{a}_{i j}^{\beta_{i}} x_{j} \leq \Psi_{\bar{b}_{i}^{\beta_{i}}}^{-1}\left(1-p_{i}\right)$ where $\Psi_{\bar{b}_{i}^{\beta_{i}}}$ is the cumulative distribution of $\bar{b}_{i}^{\beta_{i}}$. Replac$\operatorname{ing} P\left(\omega: \sum_{j=1}^{n} \underline{a}_{i j}^{\beta_{i}} x_{j} \leq \bar{b}_{i}^{\beta_{i}}(\omega)\right) \geq p_{i}$ by $\sum_{j=1}^{n} \underline{a}_{i j}^{\beta_{i}} x_{j} \leq \Psi_{\bar{b}_{i}^{\beta_{i}}}^{-1}\left(1-p_{i}\right)$ in $\mathcal{X}_{p}^{i}\left(p_{i}, \beta_{i}\right)$, we can easily see that $\forall \beta_{i} \in(0,1]$ and $\forall p_{i} \in[0,1]: X_{p}^{i}\left(p_{i}, \beta_{i}\right)$ is convex for all probability distributions of $\bar{b}_{i}^{\beta_{i}}$.

The proof is the same for the two other feasible sets; it is enough to replace, in this proof:

- $\underline{a}_{i j}^{\beta_{i}}$ and $\bar{b}_{i}^{\beta_{i}}(\omega)$ by $\bar{a}_{i j}^{1-\beta_{i}}$ and $\underline{b}_{i}^{1-\beta_{i}}(\omega)$, respectively, for $\mathcal{X}_{n}^{i}\left(p_{i}, \beta_{i}\right)$. 
- $\quad \underline{a}_{i j}^{\beta_{i}}$ and $\bar{b}_{i}^{\beta_{i}}(\omega)$ by $\sigma\left(\tilde{a}_{i j}\right)$ and $\sigma\left(\tilde{b}_{i}(\omega)\right)$, respectively, for $\mathcal{X}_{\sigma}^{i}\left(p_{i}\right)$.

These results still hold for the case of fuzzy intervals of type $L-R$.

Corollary 4 If $\tilde{a}_{i j}$ are fuzzy intervals of type $L-R$ and $\tilde{b}_{i}(\omega)$ are fuzzy random variables of type $L-R$, then $\forall \beta_{i} \in(0,1]$ and $\forall p_{i} \in[0,1]$, the feasible sets $\mathcal{X}_{p}^{i}\left(p_{i}, \beta_{i}\right), \mathcal{X}_{n}^{i}\left(p_{i}, \beta_{i}\right)$ are convex for all probability distributions of $\bar{b}_{i}$ and $\underline{b}_{i}$.

Proof $x \in \mathcal{X}_{p}^{i}\left(p_{i}, \beta_{i}\right) \Longleftrightarrow P\left\{\omega: \sum_{j=1}^{n}\left(\underline{a}_{i j}(\omega)-L^{-1}\left(\beta_{i}\right) \delta_{i j}^{a}\right) x_{j} \leq \bar{b}_{i}(\omega)+\right.$ $\left.R^{-1}\left(\beta_{i}\right) \gamma_{i}^{b}\right\} \geq p_{i}$ and we have $P\left\{\omega: \sum_{j=1}^{n}\left(\underline{a}_{i j}(\omega)-L^{-1}\left(\beta_{i}\right) \delta_{i j}^{a}\right) x_{j} \leq \bar{b}_{i}(\omega)+\right.$ $\left.R^{-1}\left(\beta_{i}\right) \gamma_{i}^{b}\right\}=1-P\left\{\omega: \sum_{j=1}^{n}\left(\underline{a}_{i j}(\omega)-L^{-1}\left(\beta_{i}\right) \delta_{i j}^{a}\right) x_{j}-R^{-1}\left(\beta_{i}\right) \gamma_{i}^{b} \geq \bar{b}_{i}(\omega)\right\}=$ $\left.1-\Psi_{\bar{b}_{i}}\left(\sum_{j=1}^{n}\left(\underline{a}_{i j}(\omega)-L^{-1}\left(\beta_{i}\right) \delta_{i j}^{a}\right) x_{j}-R^{-1}\left(\beta_{i}\right) \gamma_{i}^{b}\right\}\right)$.

Thus, $x \in \mathcal{X}_{p}^{i}\left(p_{i}, \beta_{i}\right) \Longleftrightarrow \sum_{j=1}^{n}\left(\underline{a}_{i j}(\omega)-L^{-1}\left(\beta_{i}\right) \delta_{i j}^{a}\right) x_{j}-R^{-1}\left(\beta_{i}\right) \gamma_{i}^{b} \leq \psi_{\bar{b}_{i}}^{-1}(1-$ $\left.p_{i}\right)$ where $\Psi_{\bar{b}_{i}}$ is the cumulative distribution of $\bar{b}_{i}$. We can easily see that $\forall \beta_{i} \in(0,1]$ and $\forall p_{i} \in[0,1], \mathcal{X}_{p}^{i}\left(p_{i}, \beta_{i}\right)$ is convex for all probability distributions of $\bar{b}_{i}$.

The proof is the same for the feasible set $\mathcal{X}_{n}^{i}\left(p_{i}, \beta_{i}\right)$; it is enough to replace $\underline{a}_{i j}-$ $L^{-1}\left(\beta_{i}\right) \delta_{i j}^{a}$ and $\bar{b}_{i}(\omega)+R^{-1}\left(\beta_{i}\right) \gamma_{i}^{b}$ by $\bar{a}_{i j}+R^{-1}\left(1-\beta_{i}\right) \gamma_{i j}^{a}$ and $\underline{b}_{i}(\omega)-L^{-1}\left(1-\beta_{i}\right) \delta_{i}^{b}$.

Proposition 8 If $\tilde{a}_{i j}$ are fuzzy intervals of type $L-R$ and $\tilde{b}_{i}(\omega)$ are fuzzy random variables of type $L-R$, then $\forall p_{i} \in[0,1]$, the feasible sets $\mathcal{X}_{\mu_{2 D}}^{i}\left(p_{i}, \beta_{i}\right)$ and $\mathcal{X}_{\mu_{3 D}}^{i}\left(p_{i}, \beta_{i}\right)$ are convex for all probability distributions of $\bar{b}_{i}$ and $\underline{b}_{i}$, and $\beta_{i} \geq \frac{1}{2}$.

Proof The proof is the same as the one of the previous Corollary; it is enough to replace:

- $\underline{a}_{i j}-L^{-1}\left(\beta_{i}\right) \delta_{i j}^{a}$ and $\bar{b}_{i}(\omega)+R^{-1}\left(\beta_{i}\right) \gamma_{i}^{b}$ by $\underline{a}_{i j}-L^{-1}\left(\beta_{i}\right) \delta_{i j}^{a}$ and $\underline{b}_{i}(\omega)-$ $L^{-1}\left(\beta_{i}\right) \delta_{i}^{b}$, respectively for $\mathcal{X}_{\mu_{2 D}}^{i}\left(p_{i}, \beta_{i}\right)$.

- $\underline{a}_{i j}-L^{-1}\left(\beta_{i}\right) \delta_{i j}^{a}$ and $\bar{b}_{i}(\omega)+R^{-1}\left(\beta_{i}\right) \gamma_{i}^{b}$ by $\bar{a}_{i j}+R^{-1}\left(\beta_{i}\right) \gamma_{i j}^{a}$, and $\bar{b}_{i}(\omega)+$ $R^{-1}\left(\beta_{i}\right) \gamma_{i}^{b}$, respectively for $\mathcal{X}_{\mu_{3 D}}^{i}\left(p_{i}, \beta_{i}\right)$.

5.2 Subcases where $A$ is stochastic or fuzzy stochastic

We consider the more general sub-case where both $A$ and $b$ are fuzzy stochastic first assuming, the components of $A$ and $b$ are fuzzy random variables. And then when they are fuzzy random variables of type $L-R$. Based on the previous Sect. 4, the expression of feasible sets, and Theorem 5 of Appendix B, we distinguish the case of normal fuzzy random variables (resp. of type $L-R$ ) and discrete fuzzy random variables (resp. of type $L-R$ ) and we establish the convexity of the corresponding feasible sets as follows:

\subsubsection{The components of $A$ and $b$ are fuzzy random variables}

- Case of normal fuzzy random variables 
Theorem 3 If the components of the matrix $A(m \times n)$ and the vector $b(m \times$ 1), $\tilde{a}_{i 1}, \tilde{a}_{i 2}, \ldots, \tilde{a}_{i n}$, and $\tilde{b}_{i}$, respectively, are normal fuzzy random variables whose means $\tilde{\mu}_{i 1}, \tilde{\mu}_{i 2}, \ldots, \tilde{\mu}_{i n}, \tilde{\lambda}_{i}$ are fuzzy intervals and whose variances $\sigma_{i 1}^{2}, \sigma_{i 2}^{2}, \ldots, \sigma_{i n}^{2}, \delta_{i}^{2}$, respectively are precise, then $\forall \beta_{i} \in(0,1]$ and for $p_{i}>\frac{1}{2}$, the feasible sets $\mathcal{X}_{p}^{i}\left(p_{i}, \beta_{i}\right)$ and $\mathcal{X}_{n}^{i}\left(p_{i}, \beta_{i}\right)$ are convex.

Proof From Sect. 2, item 2, on the one hand, $\underline{a}_{i 1}^{\beta_{i}}, \underline{a}_{i 2}^{\beta_{i}}, \ldots, \underline{a}_{i n}^{\beta_{i}}, \bar{b}_{i}^{\beta_{i}}$ are normal random variables with means $\underline{\mu}_{i 1}^{\beta_{i}}, \underline{\mu}_{i 2}^{\beta_{i}}, \ldots, \underline{\mu}_{i n}^{\beta_{i}}, \bar{\lambda}_{i}^{\beta_{i}}$ and precise variances $\sigma_{i 1}^{2}, \sigma_{i 2}^{2}, \ldots, \sigma_{i n}^{2}, \delta_{i}^{2}$, respectively. And on the other hand, $\bar{a}_{i 1}^{1-\beta_{i}}, \bar{a}_{i 2}^{1-\beta_{i}}, \ldots$, $\bar{a}_{i n}^{1-\beta_{i}}, \underline{b}_{i}^{1-\beta_{i}}$ are normal random variables with means $\bar{\mu}_{i 1}^{1-\beta_{i}}, \bar{\mu}_{i 2}^{1-\beta_{i}}, \ldots, \bar{\mu}_{i n}^{1-\beta_{i}}$, $\underline{\lambda}_{i}^{1-\beta_{i}}$ and precise variances $\sigma_{i 1}^{2}, \sigma_{i 2}^{2}, \ldots, \sigma_{i n}^{2}, \delta_{i}^{2}$, respectively.

Then from Theorem 5 in Appendix B, for $\forall \beta_{i} \in(0,1]$ and for $p_{i}>\frac{1}{2}$, the feasible sets $\mathcal{X}_{p}^{i}\left(p_{i}, \beta_{i}\right)$ and $\mathcal{X}_{n}^{i}\left(p_{i}, \beta_{i}\right)$ are convex.

- Case of discrete fuzzy random variables

Theorem 4 Let $\Omega$ be a finite space with probability distribution $P\left(\omega_{k}\right)=q_{k}, k=$ $1,2, \ldots, r$ and $\sum_{k=1}^{k=r} q_{k}=1$. If $a_{i 1}, a_{i 2}, \ldots, a_{i n}, b_{i}$ are $n+1$ discrete random variables based on $\Omega$, then $\forall \beta_{i} \in(0,1]$ and for $p_{i}>1-\min _{k \in(1,2, \ldots, r)} q_{k}$, the feasible sets $\mathcal{X}_{p}^{i}\left(p_{i}, \beta_{i}\right), \mathcal{X}_{n}^{i}\left(p_{i}, \beta_{i}\right)$ and $\mathcal{X}_{\sigma}^{i}\left(p_{i}\right)$ are convex.

Proof Let $\tilde{a}_{i j}(\omega)$ and $\tilde{b}_{i}(\omega)$ be discrete fuzzy random variables. Then, for $k \in$ $\{1,2, \ldots, r\}, P\left(\tilde{a}_{i j}\left(\omega_{k}\right)=\tilde{\theta}_{i j k}\right)=P\left(\tilde{b}_{i}\left(\omega_{k}\right)=\tilde{\eta}_{i k}\right)=q_{k}$, where $\tilde{\theta}_{i j k}$ and $\tilde{\eta}_{i k}$ are fuzzy intervals.

Then $\underline{a}_{i j}^{\beta_{i}}(\omega), \bar{b}_{i}^{\beta_{i}}(\omega), \bar{a}_{i j}^{1-\beta_{i}}(\omega)$ and $\underline{b}_{i}^{1-\beta_{i}}(\omega)$ are discrete random variables such that: $P\left(\underline{a}_{i j}^{\beta_{i}}\left(\omega_{k}\right)=\underline{\theta}_{i j k}^{\beta_{i}}\right)=P\left(\bar{b}_{i}^{\beta_{i}}\left(\omega_{k}\right)=\bar{\eta}_{i k}^{\beta_{i}}\right)=P\left(\bar{a}_{i j}^{1-\beta_{i}}\left(\omega_{k}\right)=\bar{\theta}_{i j k}^{1-\beta_{i}}\right)=$ $P\left(\underline{b}_{i}^{1-\beta_{i}}\left(\omega_{k}\right)=\underline{\eta}_{i k}^{1-\beta_{i}}=p_{k}\right.$, where $\underline{\theta}_{i j k}^{\beta_{i}}$ and $\bar{\eta}_{i k}^{\beta_{i}}$ are, respectively the lower and upper bounds of the $\beta_{i}$-cut of the corresponding $\tilde{\theta}_{i j k}$ and $\tilde{\eta}_{i k}$. And $\bar{\theta}_{i j k}^{1-\beta_{i}}$ and $\underline{\eta}_{i k}^{1-\beta_{i}}$ are, respectively the lower and upper bounds of the $\left(1-\beta_{i}\right)$-cut of the corresponding $\tilde{\theta}_{i j k}$ and $\tilde{\eta}_{i k}$.

In addition, $\sigma\left(\tilde{a}_{i j}(\omega)\right)$ and $\sigma\left(\tilde{b}_{i}(\omega)\right)$ are discrete real random variables such that $P\left(\sigma\left(\tilde{a}_{i j}\left(\omega_{k}\right)\right)=\sigma\left(\tilde{\theta}_{i j k}\right)\right)=q_{k}$ and $P\left(\sigma\left(\tilde{b}_{i}\left(\omega_{k}\right)\right)=\sigma\left(\tilde{\eta}_{i k}\right)\right)=q_{k}$, where $\sigma\left(\tilde{\theta}_{i j k}\right)$ and $\sigma\left(\tilde{\eta}_{i k}\right)$ are real numbers.

Consequently, from Theorem 5, in Appendix B, we conclude that: $\forall \beta_{i} \in(0,1]$ and for $p_{i}>1-\min _{k \in(1,2, \ldots, r)} q_{k}$, the feasible sets $\mathcal{X}_{p}^{i}\left(p_{i}, \beta_{i}\right), \mathcal{X}_{n}^{i}\left(p_{i}, \beta_{i}\right)$ and $\mathcal{X}_{\sigma}^{i}\left(p_{i}\right)$ are convex.

\subsubsection{The components of $A$ and $b$ are fuzzy random variables of type $L-R$}

- Case of normal fuzzy random variables of type $L-R$

Corollary 5 Let $(\Omega, F, P)$ be a probability space and $\tilde{a}_{i j}=\left(\underline{a}_{i j}, \bar{a}_{i j}, \delta_{i j}^{a}, \gamma_{i j}^{a}\right)$ and $\tilde{b}_{i}(\omega)=\left(\underline{b}_{i}(\omega), \bar{b}_{i}(\omega), \delta_{i}^{b}, \gamma_{i}^{b}\right)$ be normal fuzzy random variables of type $L-R$ such that: 
1. $\underline{a}_{i 1}, \underline{a}_{i 2}, \ldots, \underline{a}_{i n}, \underline{b}_{i}$ are normal random variables with means $\underline{\mu}_{i 1}, \underline{\mu}_{i 2}, \ldots$, $\underline{\mu}_{i n}, \underline{\lambda}_{i}$ and variances $\sigma_{i 1}^{2}, \sigma_{i 2}^{2}, \ldots, \sigma_{i n}^{2}, \delta_{i}^{2}$, respectively.

2. $\bar{a}_{i 1}, \bar{a}_{i 2}, \ldots, \bar{a}_{i n}, \bar{b}_{i}$ are normal random variables with means $\bar{\mu}_{i 1}, \bar{\mu}_{i 2}, \ldots, \bar{\mu}_{i n}$, $\bar{\lambda}_{i}$ and variances $\sigma_{i 1}^{2}, \sigma_{i 2}^{2}, \ldots, \sigma_{i n}^{2}, \delta_{i}^{2}$, respectively.

Then for $p_{i}>\frac{1}{2}$ :

- $\mathcal{X}_{p}^{i}\left(p_{i}, \beta_{i}\right)$ and $\mathcal{X}_{n}^{i}\left(p_{i}, \beta_{i}\right)$ are convex $\forall \beta_{i} \in(0,1]$.

- $\mathcal{X}_{\mu_{2 D}}^{i}\left(p_{i}, \beta_{i}\right)$ and $\mathcal{X}_{\mu_{3 D}}^{i}\left(p_{i}, \frac{1}{2}\right)$ are convex.

Proof This is a particular case of Theorem 3, as $\tilde{a}_{i j}(\omega)=\left(\underline{a}_{i j}(\omega), \bar{a}_{i j}(\omega), \delta_{i j}^{a}, \gamma_{i j}^{a}\right)$ and $\tilde{b}_{i}(\omega)=\left(\underline{b}_{i}(\omega), \bar{b}_{i}(\omega), \delta_{i}^{b}, \gamma_{i}^{b}\right)$ are normal fuzzy random variables of type $L-R$, thus, we only make the specific calculations explicit:

1. on the one hand, $\bar{b}_{i}^{\beta_{i}}=\bar{b}_{i}+R^{-1}\left(\beta_{i}\right) \gamma_{i}^{b}$ is a normal real random variable with mean $\bar{\lambda}_{i}^{\beta_{i}}=\bar{\lambda}_{i}+R^{-1}\left(\beta_{i}\right) \gamma_{i}^{b}$ and variance $\delta_{i}^{2}$ and for $j=1,2, \ldots, n: \underline{a}_{i j}^{\beta_{i}}=\underline{a}_{i j}-$ $L^{-1}\left(\beta_{i}\right) \delta_{i j}^{a}$ are normal real random variables with means $\underline{\mu}_{i j}^{\beta_{i}}=\underline{\mu}_{i j}-L^{-1}\left(\beta_{i}\right) \delta_{i j}^{a}$ and variances $\sigma_{i j}^{2}$.

2. And on the other hand, $\underline{b}_{i}^{1-\beta_{i}}=\underline{b}_{i}-L^{-1}\left(1-\beta_{i}\right) \delta_{i}^{b}$ is a normal real random variable with mean $\underline{\lambda}_{i}^{1-\beta_{i}}=\underline{\lambda}_{i}-L^{-1}\left(1-\beta_{i}\right) \delta_{i}^{b}$ and variance $\delta_{i}^{2}$ and for $j=$ $1,2, \ldots, n: \bar{a}_{i j}^{1-\beta_{i}}=\bar{a}_{i j}+R^{-1}\left(1-\beta_{i}\right) \gamma_{i j}^{a}$ are normal real random variables with means $\bar{\mu}_{i j}^{1-\beta_{i}}=\bar{\mu}_{i j}+R^{-1}\left(1-\beta_{i}\right) \gamma_{i j}^{a}$ and variances $\sigma_{i j}^{2}$.

Then we conclude that $\forall \beta_{i} \in(0,1]$ and for $p_{i}>\frac{1}{2}$, the feasible sets $\mathcal{X}_{p}^{i}\left(p_{i}, \beta_{i}\right), \mathcal{X}_{n}^{i}\left(p_{i}, \beta_{i}\right)$ are convex.

3. By replacing, in the proof of Theorem $3, \bar{b}_{i}^{\beta_{i}}$ by $\underline{b}_{i}^{\beta_{i}}$, thus $\bar{\lambda}_{i}^{\beta_{i}}$ by $\underline{\lambda}_{i}^{\beta_{i}}$ on the one hand. And on the other hand $\underline{b}_{i}^{1-\beta_{i}}$ by $\bar{b}_{i}^{1-\beta_{i}}$, thus $\underline{\lambda}_{i}^{1-\beta_{i}}$ by $\bar{\lambda}_{i}^{1-\beta_{i}}$ and taking account of the $L-R$ particularity of $\tilde{a}_{i j}$ and $\tilde{b}_{i}$, i.e. $\left(\bar{b}_{i}^{1-\beta_{i}}=\bar{b}_{i}+R^{-1}\left(1-\beta_{i}\right) \gamma_{i}^{b}\right.$, $\bar{\lambda}_{i}^{1-\beta_{i}}=\bar{\lambda}_{i}+R^{-1}\left(1-\beta_{i}\right) \gamma_{i}^{b}, \quad \bar{a}_{i j}^{1-\beta_{i}}=\bar{a}_{i j}+R^{-1}\left(1-\beta_{i}\right) \gamma_{i j}^{a}, \quad \bar{\mu}_{i j}^{1-\beta_{i}}=$ $\bar{\mu}_{i j}+R^{-1}\left(1-\beta_{i}\right) \gamma_{i j}^{a}, \quad \underline{b}_{i}^{\beta_{i}}=\underline{b}_{i}-L^{-1}\left(\beta_{i}\right) \delta_{i}^{b}, \quad \underline{\lambda}_{i}^{\beta_{i}}=\underline{\lambda}_{i}-L^{-1}\left(\beta_{i}\right) \delta_{i}^{b}, \quad \underline{a}_{i j}^{\beta_{i}}=$ $\left.\underline{a}_{i j}-L^{-1}\left(\beta_{i}\right) \delta_{i j}^{a}, \quad \underline{\mu}_{i j}^{\beta_{i}}=\underline{\mu}_{i j}-L^{-1}\left(\beta_{i}\right) \delta_{i j}^{a}.\right)$

We conclude that for $p_{i}>\frac{1}{2}$, the feasible sets $\mathcal{X}_{\mu_{2 D}}^{i}\left(p_{i}, \beta_{i}\right)$ and $\mathcal{X}_{\mu_{3 D}}^{i}\left(p_{i}, \beta_{i}\right)$ are convex $\forall \beta_{i} \geq \frac{1}{2}$.

- Case of discrete fuzzy random variables of type $L-R$

We again specialize the previous result using the additional shape assumption for fuzzy intervals.

Corollary 6 Let $\Omega$ be a finite space with probability distribution $P\left(\omega_{k}\right)=q_{k}, k=$ $1,2, \ldots, r$ and $\sum_{k=1}^{k=r} q_{k}=1$, and $a_{i 1}, a_{i 2}, \ldots, a_{i n}, b_{i}$ are $n+1$ discrete random variables based on $\Omega$. Let $\tilde{a}_{i j}=\left(\underline{a}_{i j}, \bar{a}_{i j}, \delta_{i j}^{a}, \gamma_{i j}^{a}\right)$ and $\tilde{b}_{i}(\omega)=\left(\underline{b}_{i}(\omega), \bar{b}_{i}(\omega), \delta_{i}^{b}, \gamma_{i}^{b}\right)$ be discrete fuzzy random variables of the type $L-R$. Then for $p_{i}>1-\min _{k \in(1,2, \ldots, r)} q_{k}$ : 
- $\mathcal{X}_{p}^{i}\left(p_{i}, \beta_{i}\right)$ and $\mathcal{X}_{n}^{i}\left(p_{i}, \beta_{i}\right)$ are convex $\forall \beta_{i} \in(0,1)$.

- $\mathcal{X}_{\mu_{2 D}}^{i}\left(p_{i}, \beta_{i}\right)$ and $\mathcal{X}_{\mu_{3 D}}^{i}\left(p_{i}, \beta_{i}\right)$ are convex for $\beta_{i} \geq \frac{1}{2}$.

Proof Since $\tilde{a}_{i j}=\left(\underline{a}_{i j}, \bar{a}_{i j}, \delta_{i j}^{a}, \gamma_{i j}^{a}\right)$ and $\tilde{b}_{i}(\omega)=\left(\underline{b}_{i}(\omega), \bar{b}_{i}(\omega), \delta_{i}^{b}, \gamma_{i}^{b}\right)$ are discrete fuzzy random variables of the type $L-R$, thus $\underline{a}_{i j}(\omega), \bar{a}_{i j}(\omega), \underline{b}_{i}(\omega)$ and $\bar{b}_{i}(\omega)$ are real discrete random variables. then it is obvious that: $\underline{a}_{i j}(\omega)-L^{-1}\left(\beta_{i}\right) \delta_{i j}^{a}, \bar{a}_{i j}(\omega)+$ $R^{-1}\left(1-\beta_{i}\right) \gamma_{i j}^{a}, \underline{b}_{i}(\omega)-L^{-1}\left(1-\beta_{i}\right) \delta_{i}^{b}, \underline{b}_{i}(\omega)-L^{-1}\left(\beta_{i}\right) \delta_{i}^{b}, \bar{b}_{i}(\omega)+R^{-1}\left(\beta_{i}\right) \gamma_{i}^{b}$ and $\bar{b}_{i}(\omega)+R^{-1}\left(1-\beta_{i}\right) \gamma_{i}^{b}$ are discrete random variables.

Consequently from Theorem 5 in Appendix B, $\forall \beta_{i} \in(0,1]$ and for $p_{i}>1-$ $\min _{k \in(1,2, \ldots, r)} q_{k}$, the feasible sets $\mathcal{X}_{p}^{i}\left(p_{i}, \beta_{i}\right), \mathcal{X}_{n}^{i}\left(p_{i}, \beta_{i}\right)$, are convex, and for $\beta_{i} \geq$ $\frac{1}{2}, \mathcal{X}_{\mu_{2 D}}^{i}\left(p_{i}, \beta_{i}\right)$ and $\mathcal{X}_{\mu_{3 D}}^{i}\left(p_{i}, \beta_{i}\right)$ are convex.

\section{Example}

Consider the fuzzy stochastic linear program:

$$
\left(P_{f s}^{1}\right)^{\prime}:\left\{\begin{array}{l}
\max x_{1}+2 x_{2} \\
\tilde{a}_{11} x_{1}+\tilde{a}_{12} x_{2} \leq \tilde{b}_{1}(\omega) \\
\tilde{a}_{21} x_{1}+\tilde{a}_{22} x_{2} \leq \tilde{b}_{2}(\omega) \\
x_{1} \geq 0, x_{2} \geq 0
\end{array}\right.
$$

where $\left(\tilde{a}_{i j}\right)_{i, j=1,2}$ are fuzzy intervals with piecewise linear membership functions; $\left(\tilde{b}_{i}\right)_{i=1,2}$ are discrete fuzzy random variables with discrete probability distribution $P\left(\omega_{1}\right)=0.25, P\left(\omega_{2}\right)=0.75 ;$, letting $\Omega=\left\{\omega_{1}, \omega_{2}\right\}$.

1. Case where $\left(\tilde{a}_{i j}\right)_{i, j=1,2}$ are triangular fuzzy intervals and $\left(\tilde{b}_{i}\right)_{i=1,2}$ are discrete fuzzy random variables.

$\tilde{a}_{11}=\tilde{1}, \quad \tilde{a}_{12}=\tilde{3}$

$\tilde{a}_{21}=\tilde{2}, \quad \tilde{a}_{22}=\tilde{4}$.

$P\left(\tilde{b}_{1}\left(\omega_{1}\right)=\tilde{1}\right)=P\left(\tilde{b}_{2}\left(\omega_{1}\right)=\tilde{2}\right)=0.25$ and

$P\left(\tilde{b}_{1}\left(\omega_{2}\right)=\tilde{3}\right)=P\left(\tilde{b}_{2}\left(\omega_{2}\right)=\tilde{4}\right)=0.75$. where, for $m=1,2,3,4, \tilde{m}$ is a fuzzy interval with membership function $\mu_{\tilde{m}}$ defined as follows:

$$
\mu_{\tilde{m}}(x)= \begin{cases}0 & x<m-1 \\ x-m+1 & m-1 \leq x<m \\ 1 & m \leq x<m+1 \\ -x+m+2 & m+1 \leq x \leq m+2 \\ 0 & x>m+2\end{cases}
$$

To solve the fuzzy stochastic program $\left(P_{f s}^{1}\right)^{\prime}$, we apply chance-constrained programming with fuzzy stochastic coefficients as follows:

- by combining probability and possibility with $p_{1}=p_{2}=0.75$ and $\beta_{1}=$ $\beta_{2}=0.8$, we have:

$P\left(\bar{b}_{1}^{0.8}\left(\omega_{1}\right)=2.2\right)=P\left(\bar{b}_{2}^{0.8}\left(\omega_{1}\right)=3.2\right)=0.25$ and 
$P\left(\bar{b}_{1}^{0.8}\left(\omega_{2}\right)=4.2\right)=P\left(\bar{b}_{2}^{0.8}\left(\omega_{2}\right)=5.2\right)=0.75$,

$\underline{a}_{11}^{0.8}=0.8, \underline{a}_{12}^{0.8}=2.8, \underline{a}_{21}^{0.8}=1.8, \underline{a}_{22}^{0.8}=3.8$.

We obtain:

$$
\left(P_{p}^{1}\right)^{\prime}:\left\{\begin{array}{l}
\max x_{1}+2 x_{2} \\
0.8 x_{1}+2.8 x_{2} \leq \Psi_{b_{1}^{0.8}}^{-1}(0.25)=2.2 \\
1.8 x_{1}+3.8 x_{2} \leq \Psi_{b_{2}^{0.8}}^{-1}(0.25)=3.2 \\
x_{1} \geq 0, x_{2} \geq 0
\end{array}\right.
$$

where $\Psi_{b_{i}}^{-1}, i=1,2$ are the inverse functions of the corresponding distribution function of $b_{i}$.

We obtain the solution $x^{0}=\left(\frac{11}{4}, 0\right)$ which is $(0.75,0.8)$ Pro-pos optimal for $\left(P_{f s}^{1}\right)^{\prime}$.

- by combining probability and necessity with $p_{1}=p_{2}=0.75$ and $\beta_{1}=$ $\beta_{2}=0.8$, we have:

$P\left(\underline{b}_{1}^{0.2}\left(\omega_{1}\right)=0.2\right)=P\left(\underline{b}_{2}^{0.2}\left(\omega_{1}\right)=1.2\right)=0.25$ and

$P\left(\underline{b}_{1}^{0.2}\left(\omega_{2}\right)=2.2\right)=P\left(\underline{b}_{2}^{0.2}\left(\omega_{2}\right)=3.2\right)=0.75$,

$\bar{a}_{11}^{0.2}=2.8, \bar{a}_{12}^{0.2}=4.8, \bar{a}_{21}^{0.2}=3.8, \bar{a}_{22}^{0.2}=5.8$.

We obtain

$$
\left(P_{n}^{1}\right)^{\prime}:\left\{\begin{array}{l}
\max x_{1}+2 x_{2} \\
2.8 x_{1}+4.8 x_{2} \leq \Psi_{b_{1}^{0.2}}^{-1}(0.25)=0.2 \\
3.8 x_{1}+5.8 x_{2} \leq \Psi_{b_{2}^{0.2}}^{-1}(0.25)=1.2 \\
x_{1} \geq 0, x_{2} \geq 0
\end{array}\right.
$$

where $\Psi_{b_{i}}^{-1}, i=1,2$ are the inverse functions of the corresponding distribution function of $b_{i}$.

We obtain the solution $x^{0}=\left(0, \frac{1}{24}\right)$ which is $(0.75,0.8)$ Pro-nec optimal for $\left(P_{f s}^{1}\right)^{\prime}$.

2. Case where $\left(\tilde{a}_{i j}\right)_{i, j=1,2}$ are trapezoidal fuzzy intervals and $\tilde{b}_{i=1,2}$ are discrete fuzzy random variables:

Let $\tilde{a}_{11}=(1,2,1,1)_{L-R}, \tilde{a}_{12}=(3,4,1,1)_{L-R}, \tilde{a}_{21}=(2,3,1,1)_{L-R}, \tilde{a}_{22}=$ $(4,5,1,1)_{L-R}$, and $\tilde{b}_{i}(\omega)=\left(\underline{b}_{i}(\omega), \bar{b}_{i}(\omega), 1,1\right), i=1,2$ such that:

$P\left(\tilde{b}_{1}\left(\omega_{1}\right)=\tilde{\gamma}_{1}^{1}\right)=P\left(\tilde{b}_{2}\left(\omega_{1}\right)=\tilde{\gamma}_{2}^{1}\right)=0.25$, and

$P\left(\tilde{b}_{1}\left(\omega_{2}\right)=\tilde{\gamma}_{1}^{2}\right)=P\left(\tilde{b}_{2}\left(\omega_{2}\right)=\tilde{\gamma}_{2}^{2}\right)=0.75$

with $\gamma_{1}^{1}=(1,2,1,1)_{L-R}, \gamma_{1}^{2}=(3,4,1,1)_{L-R}, \gamma_{2}^{1}=(2,3,1,1)_{L-R}, \gamma_{2}^{2}=$ $(4,5,1,1)_{L-R}$, where $L(x)=\max (0,1-x)$ and $L=R$.

To solve the fuzzy stochastic program $\left(P_{f s}^{1}\right)^{\prime}$, we apply chance-constrained programming with fuzzy stochastic coefficients by combining chance constrained programming and random interval comparison with $p_{1}=p_{2}=0.75$ and $\beta_{1}=$ $\beta_{2}=0.8$. We obtain: 
- by combining probability and $\mu_{2 D}$,

$$
\left(P_{\mu_{2 D}}^{1}\right)^{\prime}:\left\{\begin{array}{l}
\max x_{1}+2 x_{2} \\
0.2 x_{1}+2.2 x_{2} \leq \Psi_{1}^{-1}(0.25)=0.2 \\
1.2 x_{1}+3.2 x_{2} \leq \Psi_{2}^{-1}(0.25)=1.2 \\
x_{1} \geq 0, x_{2} \geq 0
\end{array}\right.
$$

where $\Psi_{i}^{-1}, i=1,2$ are the inverse functions of the corresponding distribution function of $\underline{b}_{i}-L^{-1}(0.2)$.

The solution is $x^{0}=(1,0)$ which is $(0.75,0.8)$ Pro $-\mu_{2 D}$ optimal for $\left(P_{f s}^{1}\right)^{\prime}$.

- by combining probability and $\mu_{3 D}$,

$$
\left(P_{\mu_{3 D}}^{1}\right)^{\prime}=\left\{\begin{array}{l}
\max x_{1}+2 x_{2} \\
2.2 x_{1}+4.2 x_{2} \leq \Phi_{1}^{-1}(0.25)=2.2 \\
3.2 x_{1}+5.2 x_{2} \leq \Phi_{2}^{-1}(0.25)=3.2 \\
x_{1} \geq 0, x_{2} \geq 0
\end{array}\right.
$$

where $\Phi_{b_{i}}^{-1}, i=1,2$ are the inverse functions of the corresponding distribution function of $\bar{b}_{i}+L^{-1}(0.8)$ (because $L=R$ ).

The solution $x^{0}=\left(0, \frac{8}{13}\right)$ which is $(0.75,0.8)$ Pro $-\mu_{3 D}$ optimal for $\left(P_{f s}^{1}\right)^{\prime}$.

\section{Conclusion}

In this paper, we have considered a fuzzy stochastic programming problem with a crisp objective function and fuzzy stochastic linear constraints, i.e. constraints involving fuzzy random variables or random variables and fuzzy intervals, in the general case, and fuzzy random variables of type $L-R$ or random variables and fuzzy intervals of type $L-R$ as a particular case. In order to convert these constraints into their deterministic equivalent, we have exploited various methods for comparing fuzzy intervals. Moreover, we have established conditions for the convexity of the feasible sets resulting from this transformation. The approach can be applied in the case where the right-hand side of constraints is fuzzy stochastic, stochastic, fuzzy, or deterministic with the same for its left-hand side. In the case where there is no fuzzy random variable in constraints, but only random variables or only fuzzy intervals, the proposed method, respectively reduces, when possibility theory comparison indices are used, to chance-constrained programming with stochastic coefficients due to Charnes and Cooper (1959) or to possibilistic programming with fuzzy coefficients due to Dubois (1987). The approach of Chanas and colleagues turns fuzzy programming with illknown constraint coefficients into a fusion of interval linear programming and chanceconstrained programming, which may coincide with a possibilistic approach in the case of comonotonic dependence.

In this paper we did not consider fuzzy random linear criteria. One reason is that the definition of optimal solutions cannot use the fuzzy interval comparison techniques right away. In the case of constraints, the left-hand side and the right-hand side of a 
linear constraint correspond to non-related quantities. However, fuzzy random solution evaluations pertaining to two crisp solutions $x$ and $x^{\prime}$ are no longer unrelated and cannot be compared by the techniques described above (see the discussion in Inuiguchi (2007)): they have to be adapted to account for such a relationship.

Other formulations of fuzzy stochastic programming are possible. One formulation of constraints with random coefficients may rely on stochastic dominance: namely, comparing cumulative distributions of both sides of the constraints, as an alternative to the chance-constrained approach that is based on statistical preference. The stochastic dominance approach to the comparison of random fuzzy intervals is described in Aiche and Dubois (2010). This would allow us to extend interval linear programming to coefficients described by p-boxes and other practical representations of uncertain quantities (Destercke et al. 2008).

\section{Appendices}

\section{Appendix A: Fuzzy intervals}

A fuzzy interval $\tilde{a}$ is a fuzzy set of real numbers characterised by its membership function $\mu_{\tilde{a}}: \mathbb{R} \longrightarrow[0,1]$, such that:

- there is at least one element $x \in \mathbb{R}$ such that $\mu_{\tilde{a}}(x)=1$.

- the fuzzy set is convex: $\mu_{\tilde{a}}\left(\lambda x_{1}+(1-\lambda) x_{2}\right) \geq \min \left(\mu_{\tilde{a}}\left(x_{1}\right), \mu_{\tilde{a}}\left(x_{2}\right)\right), \forall x_{1}, x_{2} \in \mathbb{R}$ and $\forall \lambda \in] 0,1]$.

A fuzzy interval $\tilde{a}$ is often called a fuzzy number if there is only one element $x \in \mathbb{R}$ such that $\mu_{\tilde{a}}(x)=1$. In this paper we assume that $\mu_{\tilde{a}}$ is upper semi-continuous (u.s.c.). Equivalently, the $\alpha$-cut of a fuzzy interval $\tilde{a}$ is a closed interval in $\mathbb{R}$ of the form:

$$
\tilde{a}^{\alpha}=\left\{x \in \mathbb{R}: \mu_{\tilde{a}}(x) \geq \alpha\right\}=\left[\underline{a}^{\alpha}, \bar{a}^{\alpha}\right]
$$

where $\alpha>0, \underline{a}^{\alpha}=\inf \left\{x \in \mathbb{R}: \mu_{\tilde{a}}(x) \geq \alpha\right\}$ and $\bar{a}^{\alpha}=\sup \left\{x \in \mathbb{R}: \mu_{\tilde{a}}(x) \geq \alpha\right\}$. In particular, the core of the fuzzy interval is $\tilde{a}^{1}=\left[\underline{a}^{1}, \bar{a}^{1}\right]$, denoted by $[\underline{a}, \bar{a}]$, for short. The strong $\alpha$-cut of a fuzzy interval $\tilde{a}$ is $\tilde{a}^{\bar{\alpha}}=\left\{x \in \mathbb{R}: \mu_{\tilde{a}}(x)>\alpha\right\}$ for $\alpha<1$. The support of a fuzzy interval $\tilde{a}$ is its strong 0 -cut $S(\tilde{a})=\left\{x \in \mathbb{R}: \mu_{\tilde{a}}(x)>0\right\}$.

The addition $\tilde{a} \oplus \tilde{b}$ of two fuzzy intervals is defined by its membership function:

$$
\mu_{\tilde{a} \oplus \tilde{b}}(z)=\sup _{x, y: x+y=z} \min \left(\mu_{\tilde{a}}(x), \mu_{\tilde{b}}(y)\right)
$$

the multiplication $\lambda \tilde{a}$ of a fuzzy interval by a constant $\lambda \neq 0$ is defined by its membership function:

$$
\mu_{\lambda \tilde{a}}(x)=\mu_{\tilde{a}}(x / \lambda)
$$

Moreover $0 \tilde{a}=0$. The $\alpha$-cut of fuzzy intervals $\tilde{a}$ and $\tilde{b}$ verify the following properties:

- $(\tilde{a}+\tilde{b})^{\alpha}=\tilde{a}^{\alpha}+\tilde{b}^{\alpha}$

- $(\lambda \tilde{b})^{\alpha}=\lambda \tilde{b}^{\alpha}, \lambda \in \mathbb{R}$. 
A fuzzy interval of the $L-R$ type is a fuzzy interval whose membership function $\mu_{\tilde{a}}$ is defined by: (see Dubois and Prade 1988)

$$
\mu_{\tilde{a}}(x)= \begin{cases}1 & \text { for } x \in[\underline{a}, \bar{a}] \\ L\left(\frac{\underline{a}-x}{\alpha_{a}}\right) & \text { for } x \leq \underline{a} \\ R\left(\frac{x-\bar{a}}{\beta_{a}}\right) & \text { for } x \geq \bar{a}\end{cases}
$$

Shape functions $L$ and $R$ are non-negative, defined on the positive real line $[0, \infty)$, non-increasing, and such that $L(0)=R(0)=1$. Coefficients $\alpha_{a}$ and $\beta_{a}$ are, respectively left and right spreads. Let $\mathcal{F}_{L R}(\mathbb{R})$ be a set of fuzzy intervals of type $L-R$. Then $\tilde{a} \in \mathcal{F}_{L R}(\mathbb{R})$ is denoted by

$$
\tilde{a}=\left(\underline{a}, \bar{a}, \alpha_{a}, \beta_{a}\right)_{L-R}
$$

Arithmetic operations on fuzzy intervals of the $L-R$ type are well-known:

- $\tilde{a} \oplus \tilde{b}=\left(\underline{a}+\underline{b}, \bar{a}+\bar{b}, \alpha_{a}+\alpha_{b}, \beta_{a}+\beta_{b}\right)_{L-R}$

- $\lambda \odot\left(\underline{a}, \bar{a}, \alpha_{a}, \beta_{a}\right)_{L-R}=\left(\lambda \underline{a}, \lambda \bar{a}, \lambda \alpha_{a}, \lambda \beta_{a}\right)_{L-R}$ if $\lambda>0$

Please refer to Dubois and Prade (1988, 1987a), Dubois et al. (2000) for details and bibliography on fuzzy intervals.

\section{Appendix B: Linear stochastic programming}

We recall known results on convexity of stochastic linear programs of the form:

$$
\left(P_{S}\right):\left\{\begin{array}{l}
\max \phi(x) \\
\sum_{j=1}^{n} a_{i j}(\omega) x_{j} \leq b_{i}(\omega), i=1, \ldots, m \\
x_{j} \geq 0, j=1, \ldots, n
\end{array}\right.
$$

where $\phi(x)$ is a deterministic linear objective function, $a_{i j}$ and $b_{i}$ are random variables. By applying the chance-constrained programming method due to Charnes and Cooper (1959), we obtain the following deterministic program:

$$
\left(P_{D}\right):\left\{\begin{array}{l}
\max \phi(x) \\
P\left(\left\{\omega: \sum_{j=1}^{n} a_{i j}(\omega) x_{j} \leq b_{i}(\omega)\right\}\right) \geq p_{i}, i=1, \ldots, m \\
x_{j} \geq 0, j=1, \ldots, n
\end{array}\right.
$$

Let $X^{i}\left(p_{i}\right)=\left\{x \geq 0: P\left(\left\{\omega: \sum_{j=1}^{n} a_{i j}(\omega) x_{j} \leq b_{i}(\omega)\right\}\right) \geq p_{i}\right\}, i=1, \ldots, m$ be the set of feasible solutions for $\left(P_{D}\right)$.

Theorem 5 (Kall 1978) Under the following conditions, the set of feasible solutions $X^{i}\left(p_{i}\right)$ is convex.

1. The feasible sets $X^{i}(0)$ and $X^{i}(1)$ are convex. 
2. If $a_{i j}$ are deterministic, then the feasible sets $X^{i}\left(p_{i}\right)$ is convex for all probability distributions of $b_{i}$.

3. If $a_{i 1}, a_{i 2}, \ldots, a_{i n}, b_{i}$ are $n+1$ normal random variables with means $\mu_{i 1}$, $\mu_{i 2}, \ldots, \mu_{i n}, \lambda_{i}$ and variances $\sigma_{i 1}^{2}, \sigma_{i 2}^{2}, \ldots, \sigma_{i n}^{2}, \delta_{i}^{2}$, respectively. Then: for $p_{i}>\frac{1}{2}$ the feasible set $X^{i}\left(p_{i}\right)$ is convex.

4. Let $\Omega$ be a finite space with probability distribution $P\left(\omega_{k}\right)=q_{k}, k=1,2, \ldots, r$ and $\sum_{k=1}^{k=r} q_{k}=1$. If $a_{i 1}, a_{i 2}, \ldots, a_{i n}, b_{i}$ are $n+1$ discrete random variables based on $\Omega$, then, for $p_{i}>1-\min _{k \in(1,2, \ldots, r)} q_{k}$ the feasible set $X^{i}\left(p_{i}\right)$ is convex.

\section{References}

Aiche, F. (1995). Sur l'optimisation floue stochastique, Dissertation (pp. 97-115). Algeria: University of Tizi-ouzou.

Aiche, F., \& Dubois, D. (2010). An extension of stochastic dominance to fuzzy random variables. In E. Hüllermeier et al. (Eds.), International conference on information processing and management of uncertainty in knowledge-based systems (IPMU 2010), LNAI 6178 (pp. 159-168), Dortmund (Germany): Springer.

Ammar, E. E. (2009). On fuzzy random multiobjective quadratic programming. European Journal of Operational Research, 193, 329-341.

Baas, S. M., \& Kwakernaak, H. (1977). Rating and ranking of multiple aspect alternatives using fuzzy sets. Automatica, 13, 47-58.

Chakraborty, D., Rao, K. R., \& Tiwari, R. N. (1994). Interactive decision making in mixed (fuzzy and stochastic) environment. European Journal of Operational Research, 31, 89-107.

Chanas, S., Delgado, M., Verdegay, J. L., \& Vila, M. A. (1993). Ranking fuzzy real intervals in the setting of random sets. Information Sciences, 69, 201-217.

Chanas, S., \& Nowakowski, M. (1988). Single value simulation of fuzzy variable. Fuzzy Sets and Systems, 25, 43-57.

Chanas, S., \& Zielinski, P. (1999). Ranking fuzzy intervals in the setting of random sets-further results. Information Sciences, 117, 191-200.

Charnes, A., \& Cooper, W. W. (1959). Chance-constrained programming. Management Science, 6, 73-79.

Couso, I., \& Dubois, D. (2009). On the variability of the concept of variance for fuzzy random variables. IEEE Transactions on Fuzzy Systems, 17, 1070-1080.

Couso, I., \& Sánchez, L. (2011). Upper and lower probabilities induced by a fuzzy random variable. Fuzzy Sets and Systems, 165(1), 1-23.

Dantzig, G. B. (1955). Linear programming under under uncertainty. Management Sciences, 1, 3-4.

David, H. (1963). The method of paired comparisons, Griffins statistical monographs \& courses (Vol. 12). London: Charles Griffin \& D. Ltd..

de Campos, L., \& Gonzalez Munoz, A. (1989). A subjective approach for ranking fuzzy numbers. Fuzzy Sets \& Systems, 29, 145-153.

Destercke, S., Dubois, D., \& Chojnacki, E. (2008). Unifying practical uncertainty representations Part I: Generalized p-boxes. International Journal of Approximate Reasoning, 49, 649-663; Part II: Clouds. 49, 664-677.

Dubois, D. (1987). Linear programming with fuzzy data. In J. C. Bezdek (Ed.), Analysis of Fuzzy Information, volume III, Application in Engineering and Sciences (pp. 241-263). Boca Raton, FL: CRC Press.

Dubois, D., Kerre, E., Mesiar, R., \& Prade, H. (2000). Fuzzy interval analysis. In: Dubois, D., \& Prade, H. (Eds.), Fundamentals of fuzzy sets, The Handbooks of Fuzzy Sets Series (pp. 483-581). Boston, MA: Kluwer.

Dubois, D., \& Prade, H. (1983). Ranking fuzzy numbers in the setting of possibility theory. Information Sciences, 30, 183-225.

Dubois, D., \& Prade, H. (1987a). Fuzzy numbers an overview. In J. C. Bezdek (Ed.), Analysis of fuzzy information, Vol. 2 (pp. 3-39). Boca Raton: CRC Press.

Dubois, D., \& Prade, H. (1987b). The mean value of a fuzzy number. Fuzzy Sets \& Systems, 24, 279-300. 
Dubois, D., \& Prade, H. (1988). Possibility theory. New York: Plenum Press.

Dubois, D., Prade, H., \& Sabbadin, R. (2001). Decision theoretic foundations of qualitative possibility theory. European Journal of Operational Research, 128, 459-478.

Fortemps, P., \& Roubens, M. (1996). Ranking and defuzzification methods based on area compensation. Fuzzy Sets \& Systems, 82, 319-330.

Fishburn, P. (1987). Interval orderings. New York: Wiley.

Inuiguchi, M. (2007). On possibilistic/fuzzy optimization. In Foundations of fuzzy logic and soft computing, IFSA 2007, LNCS 4529 (pp. 351-360), Cancun, Mexico: Springer.

Inuiguchi, M., Ichihashi, H., \& Kume, Y. (1992). Relationships between modality constrained programming problems and various fuzzy mathematical programming problems. Fuzzy Sets and Systems, 49, 243-259.

Inuiguchi, M., \& Ramik, J. (2000). Possibilistic linear programming: A brief review of fuzzy mathematical programming and a comparison with stochastic programming in portfolio selection problem. Fuzzy Sets \& Systems, 111, 3-28.

Iskander, M. G. (2005). A suggested approach for possibility and necessity dominance indices in stochastic fuzzy linear programming. Applied Mathematics Letters, 18, 395-399.

Kall, D. (1978). Stochastic linear programming (pp. 79-92). Berlin: Springer.

Katagiri, H., Sakawa, M., \& Ishii, H. (2004). Fuzzy random bottleneck spanning tree problems using possibility and necessity measures. European Journal of Operational Research, 152, 88-95.

Katagiri, H., Sakawa, M., Kato, K., \& Nishizaki, I. (2008). Interactive multiobjective fuzzy random linear programming: Maximization of possibility and probability. European Journal of Operational Research, 188, 330-339.

Kruse, H., \& Meyer, K. D. (1987). Statistics with vague data. Dordrecht: D. Riedel.

Kwakernaak, H. (1978). Fuzzy random variables I. Information Sciences, 15, 1-29.

Li, J., Xu, J., \& Gen, M. (2006). A class of multiobjective linear programming model with fuzzy random coefficients. Mathematical and Computer Modelling, 44, 1097-1113.

Liou, T., \& Wang, J. (1992). Ranking fuzzy numbers with integral value. Fuzzy Sets \& Systems, 50, 247-255.

Luhandjula, M. K. (1996). Fuzziness and randomness in an optimization framework. Fuzzy Sets \& Systems, 77, 291-297.

Luhandjula, M. K. (2004). Optimization under hybrid uncertainty. Fuzzy Sets \& Systems, 146, 187-203.

Luhandjula, M. K. (2006). Fuzzy stochastic linear programming: Survey and future research directionsfla. European Journal of Operational Research, 174, 1353-1367.

Luhandjula, M. K., \& Gupta M. M. (1996). On fuzzy stochastic optimization. Fuzzy Sets and Systems, $81,47-55$

Luhandjula, M. K., \& Joubert, J. W. (2010). On some optimisation models in fuzzy-stochastic environment. European Journal of Operational Research, 207, 1433-1441.

Ogura, Y., Li, S. M., \& Ralescu, D. A. (2001). Set Defuzzification and Choquet Integral. International Journal of Uncertainty, Fuzziness and Knowledge-Based Systems, 9, 1-12.

Puri, M., \& Ralescu, D. (1986). Fuzzy random variables. Journal of the Mathematics and Applications, 1114, 409-420

Qiao, Z., \& Wang, W. (1993). On solution and distribution problem of the linear programming with fuzzy random variable coefficients. Fuzzy Sets \& Systems, 58, 155-170.

Qiao, Z., Zhang, Y., \& Wang, W. (1994). On fuzzy random linear programming. Fuzzy Sets \& Systems, 65, 31649 .

Shapiro, A. F. (2009). Fuzzy random variables. Insurance: Mathematics and Economics, 44, 307-314.

Wang, X., \& Kerre, E. (2001). Reasonable properties for ordering of fuzzy quantities. Fuzzy Sets \& Systems, 118, 375-406.

Wang, W., \& Qiao, Z. (1993). Linear programming with fuzzy random variable coefficients. Fuzzy Sets \& Systems, 57, 295-311.

Yager, R. R. (1978). Ranking fuzzy subsets over the unit interval. In Proceedings of the IEEE international conference on decision and control (pp. 1435-1437).

Yager, R. R. (1980). On choosing between fuzzy subsets. Kybernetes, 9, 151-154.

Yager, R. R. (1993). Criteria for evaluating fuzzy ranking methods of the unit interval. Information Sciences, 24, 139-157.

Yazini, A. V. (1987). Fuzzy and stochastic programming. Fuzzy Sets \& Systems, 22, 171-188 